Copyright

by

Sergio Sebastian Rizo-Patron

2015 
The Thesis Committee for Sergio Sebastian Rizo-Patron Certifies that this is the approved version of the following thesis:

\section{Operational modal analysis of a rotating cantilever beam using high-speed Digital Image Correlation}

APPROVED BY

SUPERVISING COMMITTEE:

Jayant Sirohi, Supervisor

Krishnaswamy Ravi-Chandar 


\title{
Operational modal analysis of a rotating cantilever beam using high-speed Digital Image Correlation
}

\author{
by
}

Sergio Sebastian Rizo-Patron, B.S.As.E.

\author{
THESIS \\ Presented to the Faculty of the Graduate School of \\ The University of Texas at Austin \\ in Partial Fulfillment \\ of the Requirements \\ for the Degree of \\ MASTER OF SCIENCE IN ENGINEERING
}

THE UNIVERSITY OF TEXAS AT AUSTIN

December 2015 


\section{Acknowledgments}

I would like to thank my advisor, Dr. Sirohi, for inspiring me to pursue this course of research and for his guidance throughout my graduate career. I would also like to thank Daiju Uehara for his help in conducting the experiments described in this thesis and producing analytical models of the system, Chris Cameron for his advice and wisdom, and Young-Joon Choi and Jason Tran for helping move that monstrous tower. I would like to thank my parents

for their boundless support and for shaping me into the person I am today. Finally, I would like to thank my fiancée, Kimberly Nelligan, for all of her love and the happiness she brings me. 


\title{
Operational modal analysis of a rotating cantilever beam using high-speed Digital Image Correlation
}

\author{
Sergio Sebastian Rizo-Patron, M.S.E. \\ The University of Texas at Austin, 2015
}

Supervisor: Jayant Sirohi

\begin{abstract}
A novel procedure to perform an operational modal analysis on a rotating cantilever beam is described. This procedure uses Digital Image Correlation (DIC) to measure the deformation of a beam from images captured with a pair of high-speed digital cameras. Modal parameters including natural frequencies and mode shapes are determined from the deformation data through application of the Ibrahim Time Domain method. The procedure was validated on a $2 \mathrm{~m}$ diameter, Mach-scale helicopter rotor, excited by a jet of compressed air. Images of the rotor blade were captured at a sampling rate of $1000 \mathrm{~Hz}$ at rotational speeds up to 900 RPM. The out-of-plane deformation of the rotor was measured with a spatial resolution of $7.2 \mathrm{~mm}$ and an accuracy of $60 \mu \mathrm{m}$, or $0.006 \%$ of the rotor radius. The first three flap bending modes were identified at each rotational speed and compared to an analytical model of the system. It was found that the analytical model over-predicted the natural frequencies due to differing boundary conditions between the model and the
\end{abstract}


experiment, and so the analytical frequencies were scaled to the results of a rap test using traditional frequency domain analysis. The scaled analytical and experimental natural frequencies agreed to within $0.2 \%$ in the best case and $10.0 \%$ in the worst case. The experimental mode shapes were also found to closely match the analytical model. The results of this test demonstrate the ability of this procedure to determine the modal parameters of rotating cantilever beams. 


\section{Table of Contents}

Acknowledgments iv

Abstract $\quad$ v

List of Tables $\quad$ ix

List of Figures $\quad$ x

$\begin{array}{lll}\text { Chapter 1. Introduction } & 1\end{array}$

Chapter 2. Literature Review 3

2.1 Modal Analysis of Rotating Structures . . . . . . . . . . . . 3

2.2 Operating Deflection Shapes . . . . . . . . . . . . . . . . 9

2.3 Digital Image Correlation . . . . . . . . . . . . . . . . . . . . 10

2.4 Conclusions from Literature Review . . . . . . . . . . . . . . . 12

Chapter 3. Ibrahim Time Domain Method 14

3.1 Basic Formulation of the ITD Method . . . . . . . . . . . . . . 14

3.2 Noise Reduction through Identification of Computational Modes 19

3.3 Non-Symmetric Response Matrices and the Double Least Squares Method ................. . . 20

Chapter 4. Modal Analysis using the Ibrahim Time Domain Method 22

4.1 Simulated Beam Experiment . . . . . . . . . . . . . . . 22

4.2 Cantilever Beam Experiment . . . . . . . . . . . . . 26

4.2.0.1 Geometry of Test Setup . . . . . . . . . . 27

4.2.0.2 Test Article Preparation . . . . . . . . . . . 28

4.2.0.3 Camera Settings and Lighting . . . . . . . . 29

4.2.0.4 Calibration ............... . . 31 
4.2.0.5 Image Acquisition and Deformation Calculation 32

4.2.1 Formulation of the ITD Response Matrices . . . . . . . 35

4.2.2 Results of Cantilever Beam Experiment . . . . . . . . . 38

4.3 Accuracy of DIC Measurements and Modal Parameters . . . . 42

4.4 Conclusions of Preliminary Experiments . . . . . . . . . . . 43

Chapter 5. Helicopter Blade Experiment $\mathbf{4 5}$

5.1 Geometry of Test Setup . . . . . . . . . . . . . . . . 46

5.2 Test Article . . . . . . . . . . . . . . . . . 51

5.3 Camera Settings and Lighting ... . . . . . . . . . 53

5.4 Calibration ................... 54

5.5 Image Acquisition and Deformation Calculation . . . . . . . 55

5.6 DIC Settings . . . . . . . . . . . . . . 56

5.7 ITD Analysis Parameters . . . . . . . . . . . . . . 57

5.8 Assumptions and Approximations . . . . . . . . . . . . 58

$\begin{array}{lll}\text { Chapter 6. } & \text { Results and Analysis } & 60\end{array}$

6.1 DIC Deformations . . . . . . . . . . . . . . . . 60

6.2 ITD Analysis Results ................ 63

$\begin{array}{ll}\text { Chapter 7. Conclusion } & \mathbf{8 0}\end{array}$

7.1 Results Overview . . . . . . . . . . . . . . . . . 80

7.2 Research Recommendations . . . . . . . . . . . . . 81

7.3 Future Applications . . . . . . . . . . . . . . . . 84

$\begin{array}{lr}\text { Appendix } & 86\end{array}$

$\begin{array}{lll}\text { Appendix A. } & \text { Experimental Damping Ratios } & 87\end{array}$

$\begin{array}{lr}\text { Bibliography } & 89\end{array}$ 


\section{List of Tables}

4.1 Natural frequencies from ITD analysis and exact solution. . .

4.2 Cantilever beam natural frequency comparison from ITD, FFT, and analytical analyses. . . . . . . . . . . . . 38

6.1 Analytical vs. experimental natural frequencies. . . . . . . . . 63

6.2 Adjusted analytical vs. experimental natural frequencies. Analytical frequencies have been scaled to the results of the rap test. . . . . . . . . . . . . . .

A.1 Experimental damping ratios for cantilever beam experiment .

87 


\section{List of Figures}

2.1 Fan plot of first three flap bending natural frequencies. The black dashed lines correspond to one, three, and six per revolution frequencies. All frequencies are non-dimensionalized by 1800 RPM . . . . . . . . . . . . . . . . .

2.2 Flap bending mode shapes at 0 RPM and 10,000 RPM. The first plot shows the first mode shapes, the second shows the second mode shapes, and the third shows the third mode shapes. 6

4.1 Example deflections of simulated beam. Each time instant is

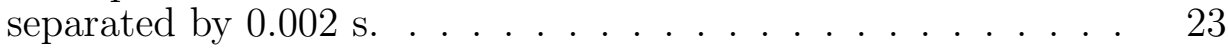

4.2 Flap bending mode shapes determined through ITD analysis. .

4.3 Percent deviation of calculated ITD mode shapes from theoretical E-B shapes. The left plot is the deviation of the first flap bending mode shape; the right plot is the deviation of the fifth flap bending mode shape. . . . . . . . . . . . .

4.4 Schematic of DIC test setup. Gray lines show field of view of each camera. Both cameras must be focused on the same region of the test article in order to find correlation between the images. 28

4.5 Cantilever beam with random speckle pattern. High-speed cameras can be seen in the foreground. Close-up of speckle pattern shown in image inset. . . . . . . . . . . . . . .

4.6 Example mask and seeding points. . . . . . . . . . . .

4.7 Example deformation field from rap test. Each box corresponds to an individual deformation vector. Red regions indicate larger deformations, and blue regions indicate smaller deformations. .

4.8 Selected deformation vectors for ITD analysis overlaid on the full range of deformation vectors calculated by DIC over the surface of the beam. . . . . . . . . . . .

4.9 Sample response at selected analysis locations. Each time in-

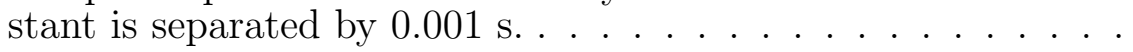

4.10 Comparison of ITD and Euler-Bernoulli mode shapes. The ITD mode shapes agree with the analytical predictions, although there is some difference as the actual beam was not perfectly cantilevered. . . . . . . . . . . . . . 
4.11 FFT of the deformation measured by DIC at the tip of the cantilever beam. The peaks circled in red correspond to the first three flap bending modes. . . . . . . . . . . . . . .

5.1 Helicopter blade experiment setup. Cameras and light fixtures are mounted to the 80/20 frame. Image capture, hydraulic motor control, and sensor monitoring is managed from inside a control room. . . . . . . . . . . . . . . .

5.2 Hall Effect sensor and piezoelectric accelerometer mounted to rotor stand. The magnet is mounted to the rotating shaft. . .

5.3 Schematic of test setup, side view. An example placement of the air nozzle is shown. . . . . . . . . . . . . . . .

5.4 Schematic of test setup, front view. An example placement of the air nozzle is shown. . . . . . . . . . . . . . . . .

5.5 Imaging sequence of blade passage. At a rotational speed of 900 RPM, five images of the blade may be captured at a rate of $1000 \mathrm{~Hz}$ before the blade moves out of the field of view. . .

5.6 Blade profile and cross sections. The blade has a VR-12 airfoil section, a chord length of $0.08 \mathrm{~m}$, and a design tip speed of 190 $\mathrm{m} / \mathrm{s} . \ldots \ldots \ldots \ldots \ldots \ldots$

5.7 Top view of rotor blade mounted to rotor stand. Close up view of speckle pattern shown in image inset. . . . . . . . . . .

5.8 Calibration setup. Calibration plate is mounted to rotor hub and $80 / 20$ frame. The plate is large enough to cover the field of view of the cameras. . . . . . . . . . . . . . . .

6.1 Excited and unexcited blade deformations at 600 RPM. . . . .

6.2 Excited blade deformations with rigid body correction at 600 RPM. . . . . . . . . . . . . . . .

6.3 Natural frequencies vs. rotational speed. Dashed lines correspond to one, five, ten, and fifteen per revolution frequencies. .

6.4 Averaged power spectrum of accelerometer response from rap tests. The first three flap bending natural frequencies are circled in red. . . . . . . . . . . . . . . . . . .

6.5 Natural frequencies vs. rotational speed. Analytical frequencies have been scaled according to the results of the rap test. Dashed lines correspond to one, five, ten, and fifteen per revolution

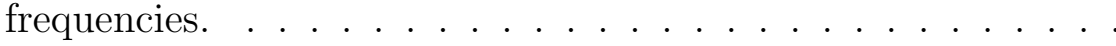

6.6 First flap bending mode shapes at 0 RPM. . . . . . . . . . 69 
6.7 First flap bending mode shapes at 300 RPM. . . . . . . . . . . 70

6.8 First flap bending mode shapes at 600 RPM. . . . . . . . . . . 70

6.9 First flap bending mode shapes at 900 RPM. . . . . . . . . . . 71

6.10 Second flap bending mode shapes. First plot is at 0 RPM, second is at $300 \mathrm{RPM}$, third is at $600 \mathrm{RPM}$, fourth is at $900 \mathrm{RPM} 72$

6.11 Third flap bending mode shapes. First plot is at 0 RPM, second is at $300 \mathrm{RPM}$, third is at $600 \mathrm{RPM}$, fourth is at $900 \mathrm{RPM}$. .

6.12 First flap bending mode shapes with increasing rotational speed. The upper plot shows the analytical mode shapes at 0,300,600, and 900 RPM. The lower plot shows the experimental mode shapes. . . . . . . . . . . . . . . 76

6.13 Second flap bending mode shapes. . . . . . . . . . . . . . . 77

6.14 Third flap bending mode shapes. . . . . . . . . . . . . . . . . 78

A.1 Experimental damping ratios for helicopter blade experiment . 88 


\section{Chapter 1}

\section{Introduction}

Operational modal analysis is the process by which the modal characteristics of a structure under operating conditions are determined. It differs from traditional modal analysis in that the input excitation to the system is generally unknown and may be random, harmonic, or both. This allows for the modal analysis of many structures for which the forcing is difficult to measure or unknowable. However, relatively few operational modal analyses have been performed on rotating structures such as helicopter blades. This is due in part to the difficulty of obtaining suitable vibration data from rotating structures. Sensors such as strain gages and accelerometers must pass signals from the rotating frame to the fixed frame using electrically noisy slip rings, and limited numbers of physical sensors may be attached to rotating structures without affecting the dynamics of the system. Additionally, the complex dynamics of rotating structures make it difficult to extract modal parameters such as natural frequencies, damping ratios, and mode shapes from this data.

This paper describes a novel procedure to determine the modal param-

eters of a rotating cantilever beam using high-speed Digital Image Correlation (DIC) and the Ibrahim Time Domain (ITD) method. This procedure cir- 
cumvents many of the challenges of performing operational modal analyses on rotating structures using traditional techniques. The procedure was experimentally validated on a $2 \mathrm{~m}$ diameter composite helicopter blade rotating at speeds up to 900 RPM. The results of the rotating experiment were then compared to an analytical model of the system. 


\section{Chapter 2}

\section{Literature Review}

\subsection{Modal Analysis of Rotating Structures}

Modal analysis of rotating structures is a growing field in structural analysis. There are many challenges associated with both the modeling and testing of rotating structures as opposed to stationary structures. When modeling rotating systems, one must account for rotational inertia, gyroscopic coupling between terms, and most importantly, centrifugal stiffening. Kane et al.[1] show that the dynamics of a rotating helicopter rotor blade can be written as,

$$
M \ddot{y}(x, t)+G(\Omega) \dot{y}(x, t)+K(\Omega) y(x, t)=f(x, t)
$$

where $y(x, t)$ is the displacement of the system at location $x$ and time $t$. This differs from a non-rotating dynamical system in that the mass $\boldsymbol{M}$ and stiffness $\boldsymbol{K}$ matrices contain terms governing the rotational inertia and centrifugal stiffening of the system. Additionally, there is a skew-symmetric gyroscopic matrix $\boldsymbol{G}$ that contains terms coupling the velocities in the directions tangent to the rotation (in-plane bending), away from the center of rotation (axial displacement), and normal to the plane of rotation (out-of-plane bending). The

stiffness matrix $\boldsymbol{K}$ and gyroscopic matrix $\boldsymbol{G}$ depend on the rotational speed, 
and therefore the eigensolution of the system is a strong function of rotational speed.

Because $\boldsymbol{G}$ is not symmetric, the solution governing the motion of the system must be determined using complex modal analysis as opposed to real modal analysis. That is, the characteristic roots of the solution will not be purely imaginary, and the mode shapes that describe the relative motion of different locations in the systems will be complex. In order to visualize the mode shapes, certain approximations must be made to transform them into real values. This is discussed further in Section 3.1. Note that damping coefficients can be included in the $\boldsymbol{G}$ matrix.

There is a vast amount of literature covering the modeling of rotating cantilever beams, including analytical models of Euler-Bernoulli beams [2], finite element analyses of Timoshenko beams [3], analyses of beams with concentrated tip masses [4], analyses of cracked rotating blades [5], and investigations of the effect of gyroscopic coupling [6].

An analysis of the relationship between rotational speed and the modal parameters of a $2 \mathrm{~m}$ diameter composite helicopter blade was performed using the University of Maryland Advanced Rotorcraft Code (UMARC). A fan plot showing the first three flap bending natural frequencies with increasing rotational speed is shown in Figure 2.1. 


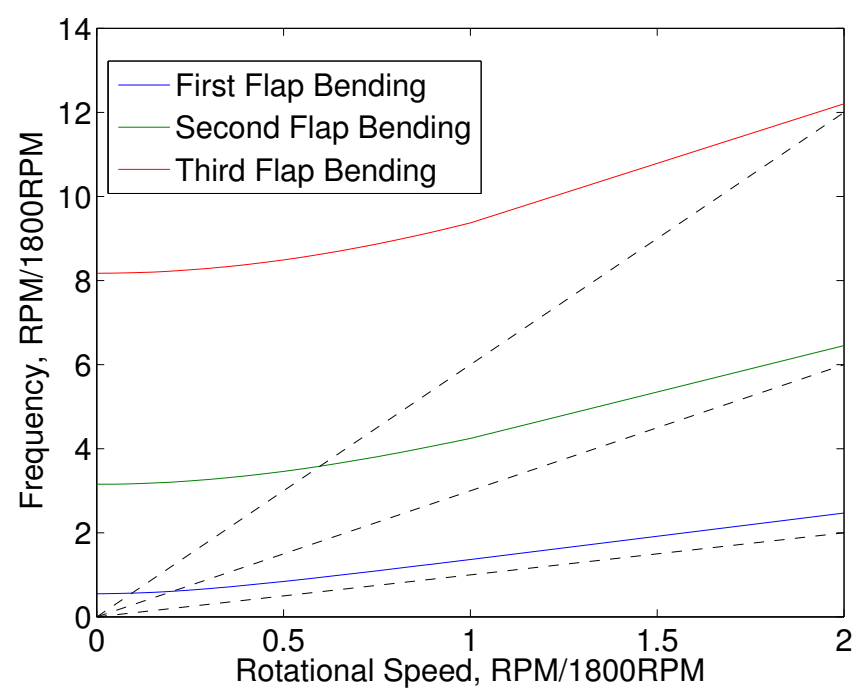

Figure 2.1: Fan plot of first three flap bending natural frequencies. The black dashed lines correspond to one, three, and six per revolution frequencies. All frequencies are non-dimensionalized by $1800 \mathrm{RPM}$

As the rotational speed increases, the blade undergoes centrifugal stiffening. This causes the flap bending natural frequencies to increase. Note that the first natural frequency approaches and then increases linearly with the rotational speed. An important result of this is that the rotational speed can never match the first flap bending natural frequency, which would cause resonance. In order to show how rotation affects the flap bending mode shapes, the first three mode shapes at 0 RPM and 10,000 RPM are presented in Figure 2.2 . 

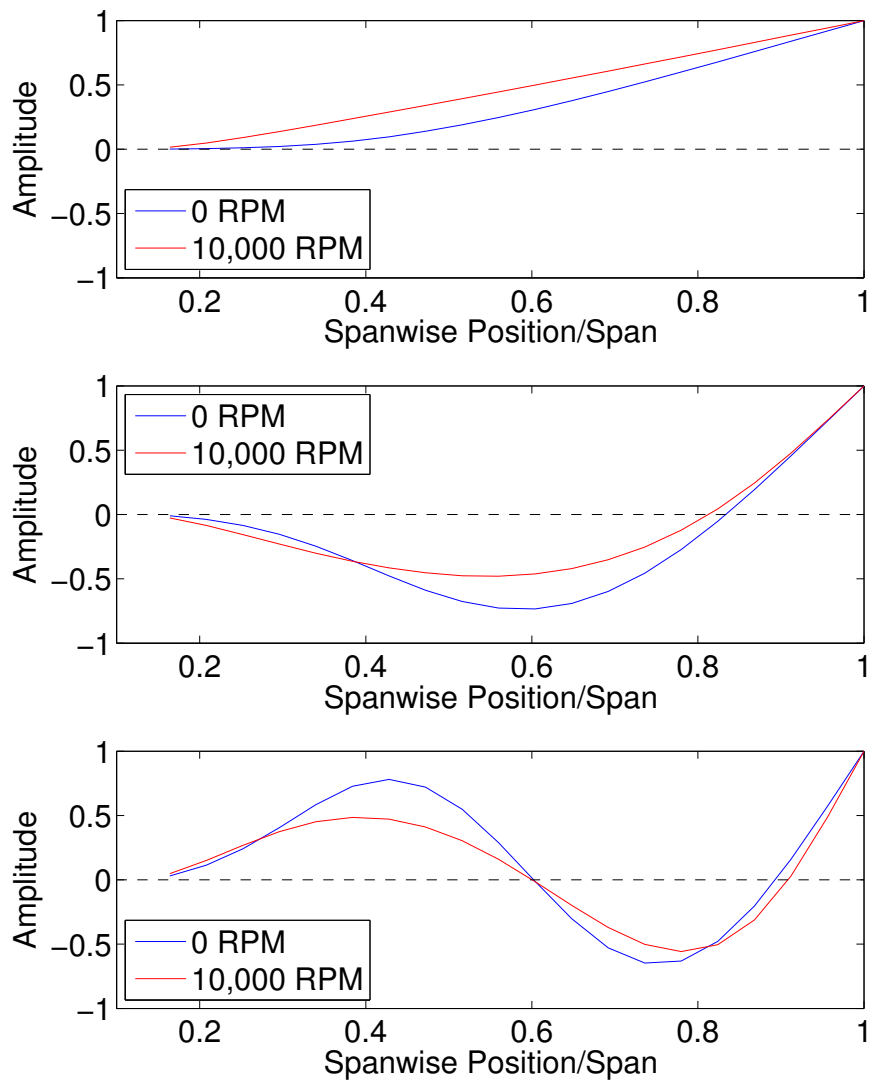

Figure 2.2: Flap bending mode shapes at 0 RPM and 10,000 RPM. The first plot shows the first mode shapes, the second shows the second mode shapes, and the third shows the third mode shapes.

The first flap bending mode shape flattens out with increasing rotational speed. The second and third shapes decrease in amplitude inboard from the tip, and the span-wise locations of the maxima and minima shift slightly. These shapes were determined at 10,000 RPM to better show the expected trends of the mode shapes, however this speed of rotation is much 
greater than the rotational speeds investigated in this study. Therefore the expected changes in mode shape will be much smaller.

According to Bucher and Ewins[7], analytical models of rotating systems may not accurately determine the actual modal parameters due to inherent uncertainties in operating boundary conditions. For example, the bearings and foundation to which a rotating system is mounted may introduce compliance at the root that is difficult to model or which changes with rotational speed. Experimental identification of modal parameters is therefore the preferred method of characterizing the response of the structure. Bucher and Ewins[7] present an overview of several techniques commonly used in experimental modal analysis of rotating structures. The primary difficulty is applying a known excitation to the system. Some examples of excitation methods include unbalancing the system with a mass attached at a specific radius, impacting the rotating shaft with an instrumented hammer, shaking the shaft with electrodynamic shakers attached though a bearing, magnetically exciting the system though active magnetic bearings, and exciting the foundation of the system. Each method has its own advantages and disadvantages, and whenever possible multiple forms of excitation are used to fully characterize the response.

Typically, the response is analyzed in the frequency domain and requires the simultaneous measurement of the system excitation. Not only can this be difficult to obtain, but because of the complex dynamics of rotating structures, it is also difficult to couple the excitation with the response to pro- 
duce frequency response functions. For example, the response may be defined in a rotating reference frame, while the excitation is defined in a stationary reference frame. Additionally, internal moving parts may induce vibration not directly caused by the applied excitation. Finally, obtaining accurate mode shapes can be very difficult because, in addition to the problems outlined above, obtaining data from enough discrete locations to properly characterize a mode shape is impractical when using conventional sensors.

While there are many numerical studies of rotating cantilever beams, there are a limited number of experimental modal analyses of rotating beams in operating conditions. Wilkie et al.[8] determined the frequencies of the first, second, and third flap-wise bending; first chord-wise bending; and first torsional modes of a 110" diameter helicopter rotor at speeds ranging from 0 to 660 RPM. These were determined from the frequency response of strain gauges attached to the rotor blade at three span-wise locations, and the signal was transmitted from the strain gauges through a 30 channel electrical slip ring. The natural frequencies were compared to theoretical values, and good correlation was found for natural frequency vs. rotor speed, especially for the first two flap bending modes. However, it was found that the analytical model over-predicted the flap bending natural frequencies. No attempt was made to determine the mode shapes due to the limited spatial resolution of the data, i.e. only three span-wise locations.

Lundstrom et al.[9] performed an operational modal analysis on the rotor blade of a Robinson R44 helicopter in hover. Similar to the present study, 
the vibration of the helicopter blade was measured using stereophotogrammetry. High-speed 3D dynamic point tracking was used to measure the motion of 22 vinyl targets mounted along the span of the rotor blade. The vibration data was analyzed in the frequency domain, and several Operating Deflection Shapes were then obtained from the vibration data. As will be discussed in the following section, while Operating Deflection Shapes are useful measurements of a system's pattern of motion at a particular operating condition, they do not necessarily correspond to the actual modal parameters of the system.

\subsection{Operating Deflection Shapes}

It is important to differentiate between Operating Deflection Shapes (ODS) and mode shapes, as this is one of the main motivations for the present study. Most operational modal analyses seek to characterize the ODS's of a structure. Schwarz and Richardson[10] define an ODS most generally as "the values of a set of time domain responses at a specific time, or the values of a set of frequency domain responses at a specific frequency." In other words, it is the pattern of motion of a structure under a particular operating condition, and it may be represented as a sum of modes. When the excitation frequency is close to a natural frequency and the ODS is dominated by one mode, the ODS will approximate a mode shape. These, however, are restrictive assumptions, and ultimately only produce estimates of the actual modal parameters, which

include natural frequencies, damping ratios, and mode shapes. As will be shown in Section 3, the ITD method of modal analysis does not approximate 
the modal parameters from ODS's, but instead directly determines them from the response of the system measured at several locations. In this study, the vibration of a cantilever beam is measured using 3D Digital Image correlation, and the modal parameters are determined though ITD analysis.

\subsection{Digital Image Correlation}

Digital image correlation is an optical deformation measurement technique that can be used to measure the whole-field deformation of a body (Kahn-Jetter and Chu[11]). A high-contrast speckle pattern is applied to the test article, and a pair of high-resolution cameras separated by a fixed distance captures images of the undeformed and deformed surface. A mapping function determined through a calibration procedure translates image coordinates into three-dimensional physical coordinates using the parallax between the two camera images. Cross-correlation of the undeformed and deformed images, in conjunction with the mapping function yields a deformation map of the surface.

Sirohi and Lawson[12] performed an experimental study on the application of DIC to measure the flapping, lead-lag, and torsional deformation of rotating helicopter blades with diameters ranging from $7.6 \mathrm{~cm}$ to $61 \mathrm{~cm}$ rotating at 1500 RPM. Images were captured at specific azimuthal positions by synchronizing image capture with a once-per-revolution trigger provided by a Hall Effect sensor, and the deformation was determined by averaging the re-

sults from 100 revolutions. It was found that out-of-plane deformations up to 
$10 \mathrm{~mm}$ could be measured with an accuracy of $97 \mu \mathrm{m}$, or $0.04 \%$ of the rotor radius, and that DIC was a viable tool for determining the full-field deformation of rotating blades.

Sicard and Sirohi[13] extended this procedure to measure the deformation of an extremely flexible $46 \mathrm{~cm}$ diameter rotor in hover in order to validate and improve aeroelastic models of the rotor blade. Images were captured of the blade rotating at $1200 \mathrm{RPM}$, and the full-field deformation was calculated with an accuracy of $15 \mu \mathrm{m}$, or $0.02 \%$ of the rotor radius. It was determined that an accurate model of this system must include flap and lead-lag bending as well as twist degrees of freedom. Additionally, it was found that the elastic twist of the blade is of the same order of magnitude as the blade root pitch. Such conclusions would be difficult to make without the benefit of DIC, as it is impossible to measure this rotor blade deformation using conventional means.

Tran et al.[14] measured the deformation of a flexible flapping wing using DIC to validate a reduced-order model developed from a POD-Galerkin projection method. The DIC results not only showed good correlation with the reduced-order model, but also with direct numerical simulation and previously published data.

These experiments demonstrate the suitability of DIC to measure the deformation of static, dynamic, and rotating systems. When used in conjunction with high-speed imagery, DIC can be used to measure the vibration of a system. This vibration data can then be analyzed to determine the modal parameters of the system. 


\subsection{Conclusions from Literature Review}

Operational modal analysis is an important part of the developmental cycle for many structures designed to withstand dynamic loading. However, there are relatively few experimental studies of the modal parameters of rotating structures and how these parameters develop with increasing rotational speeds. This is due to two reasons. First, collecting vibration data of rotating structures is challenging. Traditional sensors such as strain gauges and accelerometers must pass signals through electrically noisy slip rings, and limited numbers of physical sensors can be attached to rotating structures without affecting the dynamics of the system. Second, it is very difficult to analyze these signals using traditional frequency analysis. Developing frequency response functions requires measuring the dynamic excitation of the system, which can be even more difficult to obtain than the vibration response. Operating Deflection Shapes can be developed, but these have limited use in verifying models of the system.

The first issue can be addressed by using DIC or other stereophotogrammetic techniques. DIC allows for accurate, full-field measurements of the deformation of a rotating system without changing its operating conditions. The second issue can be resolved by analyzing the response using the ITD method, which does not require the excitation to be measured and determines modal parameters without the use of ODS's. In this thesis, a method to perform an ITD analysis using DIC measurements of a rotating cantilever beam is developed. The method is validated through an operational modal analysis of a 
rotating helicopter blade. 


\section{Chapter 3}

\section{Ibrahim Time Domain Method}

The Ibrahim Time Domain (ITD) method of modal analysis was developed as an alternative to frequency domain modal analyses (Ibrahim [15]). One of the primary benefits of this method is that it does not require measurements of the excitation of the system under investigation. This makes it an ideal candidate for Operational modal analysis, where the excitation is generally unknown. In this chapter, the basic formulation of the ITD procedure is described along with additional techniques to accurately determine the modal parameters of a system with unknown excitation.

\subsection{Basic Formulation of the ITD Method}

The free vibration of a linear system with general damping such as in Eq. (2.1) can be described by,

$$
y(x, t)=\sum \psi_{r}(x) e^{\lambda_{r} t}
$$

where $y(x, t)$ is the displacement of the system at location $x$ and time $t, \psi_{r}$ is the the system's $r^{t h}$ mode shape, and $\lambda_{r}$ is the system's $r^{\text {th }}$ characteristic root that contains the corresponding natural frequency and damping ratio. 
For undamped, non-rotating structures, the $\boldsymbol{G}$ matrix in Eq. (2.1) will be zero, and so the characteristic roots will be purely imaginary conjugates and the mode shapes will be real-valued. Actual structures posses some viscous damping however. The characteristic roots will therefore posses both real and imaginary components and the mode shapes will appear as complex conjugate pairs. In addition to viscous damping, gyroscopic coupling or measurement noise will populate the $\boldsymbol{G}$ matrix.

If one assumes $N$ modes are contributing to the response and are represented by complex conjugate pairs, the vibration can be represented as the following vector product:

$$
y(x, t)=\left\{\begin{array}{llll}
\psi_{1}(x) & \psi_{2}(x) & \cdots & \psi_{2 N}(x)
\end{array}\right\}\left\{\begin{array}{c}
e^{\lambda_{1} t} \\
e^{\lambda_{2} t} \\
\vdots \\
e^{\lambda_{2 N} t}
\end{array}\right\}
$$

where $\psi_{1}$ and $\psi_{2}$ is the conjugate pair that defines the first mode shape, and $\lambda_{1}$ and $\lambda_{2}$ is the conjugate pair that defines the first characteristic root.

The system response at $N$ different locations and $2 N$ time instants is thus defined as

$$
\begin{gathered}
\boldsymbol{y}=\left[\begin{array}{ccc}
y\left(x_{1}, t_{1}\right) & \cdots & y\left(x_{1}, t_{2 N}\right) \\
\cdots & \ddots & \cdots \\
y\left(x_{N}, t_{1}\right) & \cdots & y\left(x_{N}, t_{2 N}\right)
\end{array}\right] \\
=\left[\begin{array}{ccc}
\psi_{1}\left(x_{1}\right) & \cdots & \psi_{2 N}\left(x_{1}\right) \\
\cdots & \ddots & \cdots \\
\psi_{2 N}\left(x_{N}\right) & \cdots & \psi_{2 N}\left(x_{N}\right)
\end{array}\right]\left[\begin{array}{ccc}
e^{\lambda_{1} t_{1}} & \cdots & e^{\lambda_{1} t_{2 N}} \\
\cdots & \ddots & \cdots \\
e^{\lambda_{2 N} t_{1}} & \cdots & e^{\lambda_{2 N} t_{2 N}}
\end{array}\right]=\boldsymbol{\psi} \boldsymbol{\Lambda}
\end{gathered}
$$


It is important to note that there is no restriction on the choice of $t_{1}, t_{2}, \ldots, t_{2 N}$. Now consider the same system delayed by a time interval $\Delta t$ :

$$
\bar{y}=y(x, t+\Delta t)=\sum \psi_{r}(x) e^{\lambda_{r}(t+\Delta t)}=\sum \psi_{r}(x) e^{\lambda_{r} \Delta t} e^{\lambda_{r} t}
$$

In matrix form, this is

$$
\begin{array}{r}
\overline{\boldsymbol{y}}=\left[\begin{array}{ccc}
y\left(x_{1}, t_{1}+\Delta t\right) & \cdots & y\left(x_{1}, t_{2 N}+\Delta t\right) \\
\cdots & \ddots & \cdots \\
y\left(x_{N}, t_{1}+\Delta t\right) & \cdots & y\left(x_{N}, t_{2 N}+\Delta t\right)
\end{array}\right] \\
=\boldsymbol{\psi}\left[\begin{array}{ccc}
e^{\lambda_{1} \Delta t} & 0 & 0 \\
0 & \ddots & 0 \\
0 & 0 & e^{\lambda_{2 N} \Delta t}
\end{array}\right] \boldsymbol{\Lambda}=\boldsymbol{\psi} \boldsymbol{\alpha} \boldsymbol{\Lambda}
\end{array}
$$

Combining both the original system response and the delayed response yields a single $2 N \times 2 N$ response matrix:

$$
\left[\begin{array}{c}
y \\
\bar{y}
\end{array}\right]=\left[\begin{array}{c}
\psi \\
\psi \alpha
\end{array}\right] \Lambda
$$

or

$$
\Phi=\Psi \Lambda
$$

Applying another time shift to the system will result in a new $2 N \times 2 N$ system matrix:

$$
\left[\begin{array}{c}
\hat{y} \\
\hat{\bar{y}}
\end{array}\right]=\left[\begin{array}{c}
\psi \alpha \\
\psi \alpha^{2}
\end{array}\right] \Lambda
$$

or

$$
\hat{\boldsymbol{\Phi}}=\Psi \alpha \Lambda
$$


The two systems can now be manipulated to eliminate the matrix containing the time-dependent term, $[\Lambda]$ :

$$
\hat{\Phi} \Phi^{-1} \Psi=\alpha \Psi
$$

The corresponding eigenvalue problem is

$$
\hat{\boldsymbol{\Phi}} \Phi^{-1}\left\{\Psi_{r}\right\}=\alpha_{r}\left\{\boldsymbol{\Psi}_{r}\right\}
$$

where $\hat{\boldsymbol{\Phi}}$ and $\boldsymbol{\Phi}$ are the response matrices, $\{\boldsymbol{\Psi}\}$ is the system's $r^{\text {th }}$ mode shape, and $\alpha_{r}$ contains the corresponding natural frequency and damping ratio.

Referring back to Eq. (3.5), one can see that

$$
\alpha_{r}=e^{\lambda_{r} \Delta t}
$$

where $\lambda_{r}$ is the $r^{\text {th }}$ characteristic root of the system described in Eq. 3.1, and $\Delta t$ is the time shift between the response matrices.

For underdamped systems, the $r^{\text {th }}$ characteristic root $\lambda_{r}$ is defined as,

$$
\lambda_{r}=-\zeta_{r}\left(\omega_{n}\right)_{r} \pm\left(\omega_{n}\right)_{r} \sqrt{\zeta_{r}^{2}-1}
$$

where $\left(\omega_{n}\right)_{r}$ is the $r^{t h}$ natural frequency and $\zeta_{r}$ is the $r^{t h}$ damping ratio. The damping ratio is less than 1 for an underdamped system, so the characteristic root will be complex.

Because $\lambda_{r}$ is complex valued, $\alpha_{r}$ from Eq. (3.12) must also be complex. Let:

$$
\alpha_{r}=a_{r}+j b_{r}
$$


and

$$
\lambda_{r}=c_{r}+j d_{r}
$$

where $a_{r}$ and $b_{r}$ are the real and imaginary components of $\alpha_{r}$, and $c_{r}$ and $d_{r}$ are the real and imaginary components of $\lambda_{r}$. The real and imaginary components of the characteristic root $\lambda_{r}$ can therefore be determined as,

$$
c_{r}=\frac{1}{2 \Delta t} \ln \left(a_{r}^{2}+b_{r}^{2}\right)
$$

and

$$
d_{r}=\frac{1}{\Delta t} \tan ^{-1}\left(\frac{b_{r}}{a_{r}}\right)
$$

Referencing Eq. (3.13), one can see,

$$
c_{r}=-\zeta_{r}\left(\omega_{n}\right)_{r}
$$

and

$$
d_{r}=\left(\omega_{n}\right)_{r} \sqrt{\zeta_{r}^{2}-1}
$$

The $r^{\text {th }}$ natural frequency $\left(\omega_{n}\right)_{r}$ is therefore defined as

$$
\left(\omega_{n}\right)_{r}=\sqrt{c_{r}^{2}+d_{r}^{2}}
$$

and the $r^{\text {th }}$ damping ratio $\zeta_{r}$ is

$$
\zeta_{r}=\frac{c_{r}}{\sqrt{c_{r}^{2}+d_{r}^{2}}}
$$




\subsection{Noise Reduction through Identification of Compu- tational Modes}

Suppose the number of modes contributing to the response of the system is equal to $N$. In the basic formulation of the ITD procedure, the system response matrix $\boldsymbol{y}$ in Eq. (3.3) will be formulated using the response at $N$ locations and $2 N$ time instants. If these responses contain measurement noise, the eigenvalues and eigenvectors determined from Eq. (3.11) must represent both the structural response and the underlying noise, distorting the resultant modal characteristics. In other words, the mode shapes $\psi_{r}$ and characteristic roots $\lambda_{r}$ in Eq. (3.1) will include both the actual response and a contribution due to the measurement noise in $y(x, t)$.

If, however, the system response matrices are formulated using the response at $M$ locations and $2 M$ time instants where $M>N$, or the number of measurement locations is greater than the number of contributing modes above the noise floor, then ITD analysis will calculate more modes than are contributing to the response. These extra modes are known as computational modes, and they characterize the noise of the system.

Another way to view this is by replacing Eq. (3.1) with,

$$
y(x, t)=\sum_{r=1}^{N} \psi_{r}(x) e^{\lambda_{r} t}+n(x, t)
$$

where $n(x, t)$ is the noise added to the response. This noise can be modeled as computational modes of the same form as the structural modes:

$$
y(x, t)=\sum_{r=1}^{N} \psi_{r} e^{\lambda_{r} t}+\sum_{r=N+1}^{M} \psi_{r} e^{\lambda_{r} t}
$$


Thus $M-N$ modes produced by ITD analysis will be computational modes. These computational modes act as outlets for noise, increasing the accuracy of the $N$ structural modes.

The solution will now contain both structural and computational modes, and a method to separate them is required. Ones such method is to solve the eigenvalue problem for a different set of time instants and compare the complex eigenvectors from both solutions using the Mode Shape Correlation Constant (MSCC)[16], defined as,

$$
M S C C=\frac{\left|\left\{\gamma_{1}\right\}^{T}\left\{\gamma_{2}\right\}^{*}\right|^{2}}{\left\{\gamma_{1}\right\}^{T}\left\{\gamma_{1}\right\}^{*}\left\{\gamma_{2}\right\}^{T}\left\{\gamma_{2}\right\}^{*}}
$$

where $\left\{\gamma_{1}\right\}$ is the first modal vector, $\left\{\gamma_{2}\right\}$ is the second modal vector, and * denotes the complex conjugate. If two mode shapes have an MSCC close to $1.00+i * 0.00$ then the modes are correlated. Frequency and damping matching combined with the MSCC can be used to separate actual modes from computational modes, which will not be correlated between the two solutions.

\subsection{Non-Symmetric Response Matrices and the Double Least Squares Method}

The accuracy of the modal parameters generated by the ITD method can be further improved by formulating an overdetermined system matrix and reducing it using a least-squares approximation. The system is overdetermined if the response matrices $\hat{\boldsymbol{\Phi}}$ and $\boldsymbol{\Phi}$ in Eq. (3.11) are constructed to be of size $2 N \times R$ with $R>2 N$ corresponding to the number of time instants measured. 
The least squares solution of the system is calculated as,

$$
\boldsymbol{A}\left\{\boldsymbol{\Psi}_{r}\right\}=\alpha_{r}\left\{\boldsymbol{\Psi}_{r}\right\}
$$

where

$$
A=\left[\hat{\Phi} \Phi^{T}\right]\left[\Phi \Phi^{T}\right]^{-1}
$$

This has been shown, however, to produce large errors in the calculated damping ratios. Ibrahim[17] shows that using a double least squares approximation yields much more accurate damping ratios while preserving the accuracy of the natural frequencies and mode shapes. The system matrix $\boldsymbol{A}$ in Eq. (3.25) is calculated by the double least squares approach as

$$
A=\frac{1}{2}\left(\left[\hat{\Phi} \Phi^{T}\right]\left[\Phi \Phi^{T}\right]^{-1}+\left[\hat{\Phi} \hat{\Phi}^{T}\right]\left[\Phi \hat{\Phi}^{T}\right]^{-1}\right)
$$

Ibrahim[17] recommends keeping $R>4 N$ to ensure an accurate least squares approximation. 


\section{Chapter 4}

\section{Modal Analysis using the Ibrahim Time Domain Method}

In this chapter, the procedure to extract modal information from deformation data using the ITD method is demonstrated through two experiments. In the first experiment, the deformation of a cantilever beam is simulated using Euler-Bernoulli beam theory. In the second experiment, the deformation of an actual cantilever beam is measured using DIC.

The goals of these experiments are to demonstrate the ability of ITD to extract modal parameters from deformation data, as well as to describe the procedure to obtain deformation data using DIC. Finally, the accuracy of both the deformation measured using DIC and the modal parameters determined through ITD analysis will be discussed.

\subsection{Simulated Beam Experiment}

In order to verify the ability of the ITD method to accurately extract modal parameters, an ITD analysis was performed on a simulated cantilever beam. The vibration response of a $0.45 \times 0.05 \times 0.0005 \mathrm{~m}$ aluminum cantilever beam subjected to an initial displacement varying cubicly along its span was 
simulated using Euler-Bernoulli beam theory. The displacement at 80 locations along the span was calculated at 320 time intervals of 0.002 s. These displacements were then used to populate the response matrices $\hat{\boldsymbol{\Phi}}$ and $\boldsymbol{\Phi}$ in Eq. (3.11). The modal parameters were determined from the solution to the eigenvalue problem in Eq. (3.11). The response simulated at three time instants separated by $0.002 \mathrm{~s}$ is shown in Figure 4.1 .

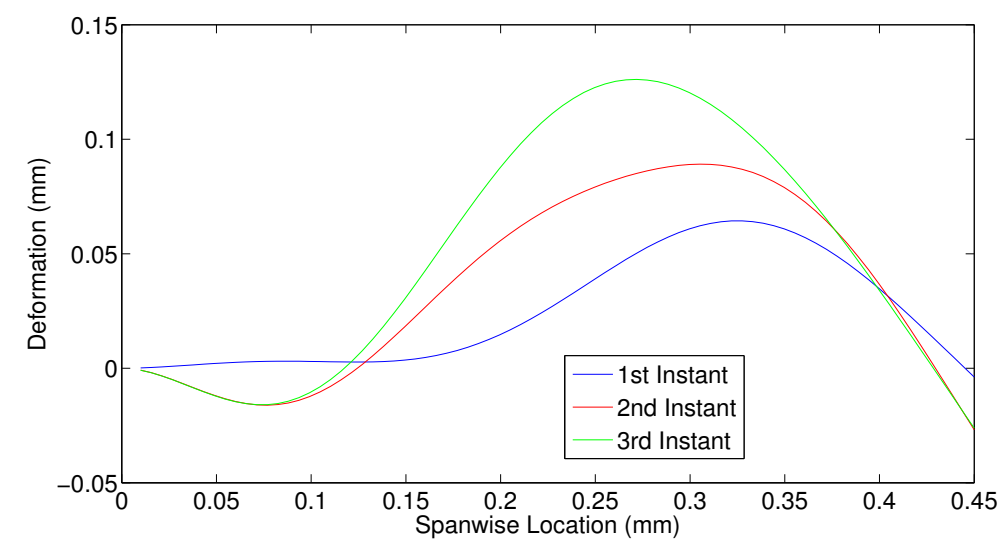

Figure 4.1: Example deflections of simulated beam. Each time instant is separated by $0.002 \mathrm{~s}$.

The mode shapes determined through ITD analysis are shown in Figure 4.2, and the percentage difference between the ITD mode shapes and the mode shapes from the Euler-Bernoulli beam theory is shown in Figure 4.3. A comparison of the Euler-Bernoulli and ITD calculated natural frequencies is shown in Table 4.1. 


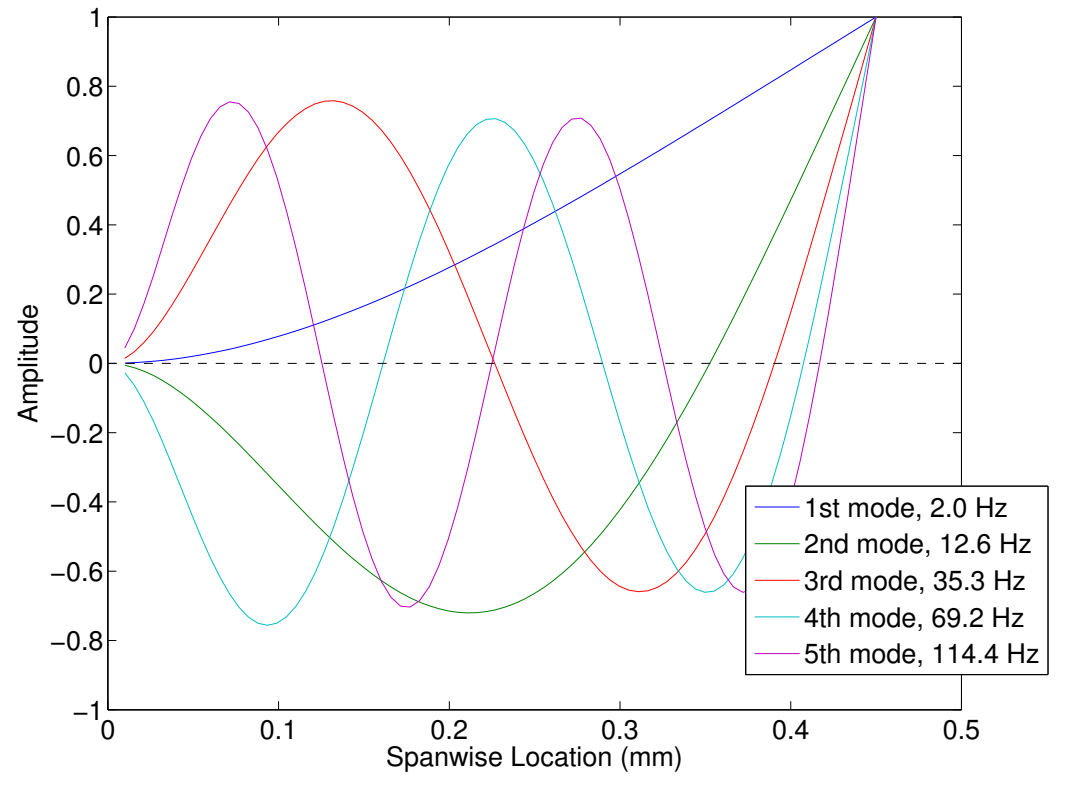

Figure 4.2: Flap bending mode shapes determined through ITD analysis.

The ITD method was able to accurately reproduce all five natural frequencies and mode shapes contributing to the response of the system. The maximum error in the measured natural frequencies is $0.002 \%$. The first mode shape was the most accurate, with a maximum deviation of $0.003 \%$ from the exact solution. The fifth mode shape was the least accurate, with a maximum deviation of $0.1 \%$. 

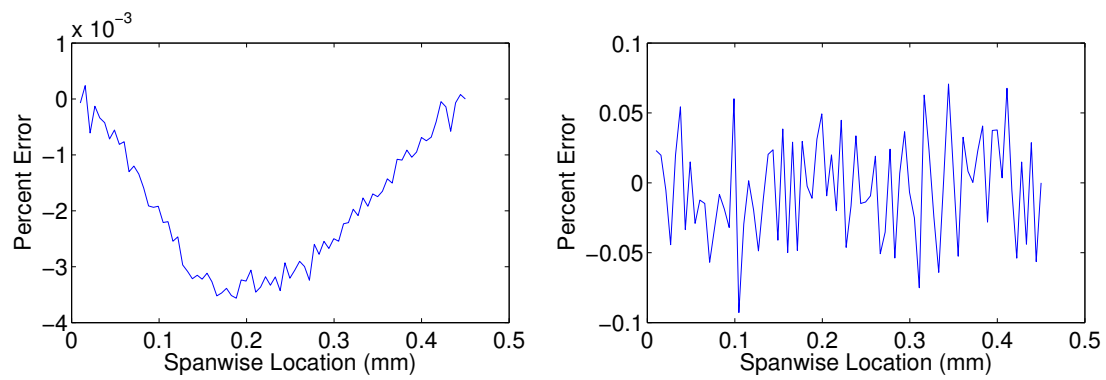

Figure 4.3: Percent deviation of calculated ITD mode shapes from theoretical E-B shapes. The left plot is the deviation of the first flap bending mode shape; the right plot is the deviation of the fifth flap bending mode shape.

\begin{tabular}{|c|c|c|c|}
\hline Mode & $\begin{array}{c}\text { ITD Frequency } \\
(\mathrm{Hz})\end{array}$ & $\begin{array}{c}\text { Exact Frequency } \\
(\mathrm{Hz})\end{array}$ & $\begin{array}{c}\text { Percent } \\
\text { Difference }\end{array}$ \\
\hline First Flap & 2.0117 & 2.0117 & $0 \%$ \\
\hline Second Flap & 12.6080 & 12.6078 & $0.002 \%$ \\
\hline Third Flap & 35.2976 & 35.2969 & $0.002 \%$ \\
\hline Fourth Flap & 69.1811 & 69.1815 & $0.001 \%$ \\
\hline Fifth Flap & 114.3598 & 114.3613 & $0.001 \%$ \\
\hline
\end{tabular}

Table 4.1: Natural frequencies from ITD analysis and exact solution. 


\subsection{Cantilever Beam Experiment}

One of the challenges of performing an ITD analysis on a structure is collecting data at a sufficient number of locations simultaneously to populate the response matrices $\hat{\boldsymbol{\Phi}}$ and $\boldsymbol{\Phi}$ in Eq. (3.11). This normally requires numerous individual strain gauges or accelerometers to be mounted to the structure. Ibrahim[15] developed a method to artificially increase the number of measurement locations by using time-shifted responses known as pseudomeasurements, however the spatial resolution of the resultant mode shapes is still limited by the number of sensors.

DIC produces deformation vectors over the whole field of interest. This not only eliminates the need for pseudo-measurements, but also allows for multiple formulations of the ITD analysis from the same set of data using different measurement locations. For example, if deformation vectors are produced across the chord and span of a beam, the response matrices can be formulated using a strip of vectors along a constant chord-wise location to produce flap bending mode shapes. The user can then formulate a new set of response matrices using a strip of vectors along a different constant chord-wise location to produce an independent set of flap bending mode shapes. The scatter of the resultant modal parameters can then be determined without requiring multiple tests. For these reasons, DIC is an excellent tool to obtain the vibration response for an ITD analysis.

The use of DIC measurements for ITD analysis is demonstrated through the modal analysis of a $0.45 \times 0.04 \times 0.0007 \mathrm{~m}$ aluminum cantilever beam 
subjected to an impulse at its midspan provided by a small hammer. Images of the vibration of the beam were captured using a pair of Phantom Miro M310 high-speed digital cameras with a $35 \mathrm{~mm}$ focal length lens. DIC was performed on the images using LaVision DaVis 8.2.2 image processing software. In this section, the procedure to obtain deformation data using DIC is described, and the results of the experiment are presented.

The spatial resolution and accuracy of DIC measurements are dependent on the test setup. This includes the geometry of the test setup, the preparation of the test article, the camera settings and lighting, the calibration procedure, and the parameters of the DIC calculation.

\subsubsection{Geometry of Test Setup}

Three dimensional surface heights, deformations, and strains of a structure are determined through DIC by capturing images of the structure with two high-resolution cameras separated by a fixed distance. The focal length of the camera lenses and the distance from the camera to the test article determine the field of view of the cameras. A longer focal length and a smaller distance between the camera and the test article will resolve smaller vibrations but decrease the field of view. It is possible to analyze several smaller sections of the test article independently and stitch together the results in

post-processing, but this increases complexity and introduces other sources of uncertainty, such as how well the images are aligned.

DIC determines the 3D position of points in the field of focus using the 
parallax between the two camera images. Thus a larger camera separation will better resolve 3D displacements, however the whole region of interest must be visible in both cameras. Additionally, if the test article undergoes large displacements, a larger camera separation will decrease the size of the region in which the test article will be simultaneously in focus for both cameras.

For this experiment, the two cameras were positioned $1 \mathrm{~m}$ from the cantilever beam with $0.5 \mathrm{~m}$ separation between the cameras. A schematic of the test setup is shown in Figure 4.4.

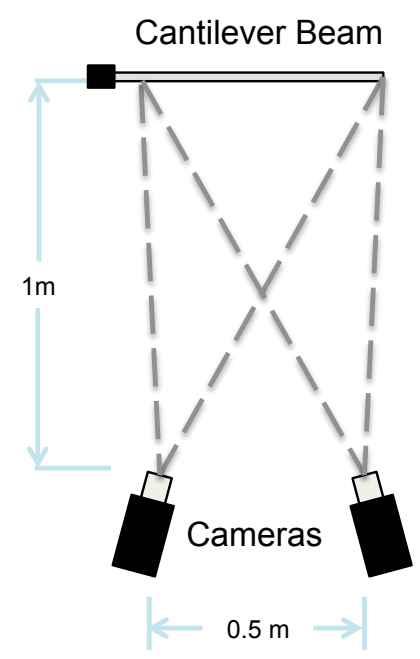

Figure 4.4: Schematic of DIC test setup. Gray lines show field of view of each camera. Both cameras must be focused on the same region of the test article in order to find correlation between the images.

\subsubsection{Test Article Preparation}

A high-contrast speckle pattern must be applied to the test article for DIC to determine the deformation of individual points. A uniform matte 
black background with white speckles or a matte white background with black speckles are suitable choices. The speckles should be sized so that they are approximately 3 pixels in diameter with a 3 to 9 pixel separation between speckles. Thus the ideal speckle size will be determined by the focal length and distance from the cameras to the test article. A picture of the test setup with a close-up of the speckle pattern is shown in Figure 4.5.

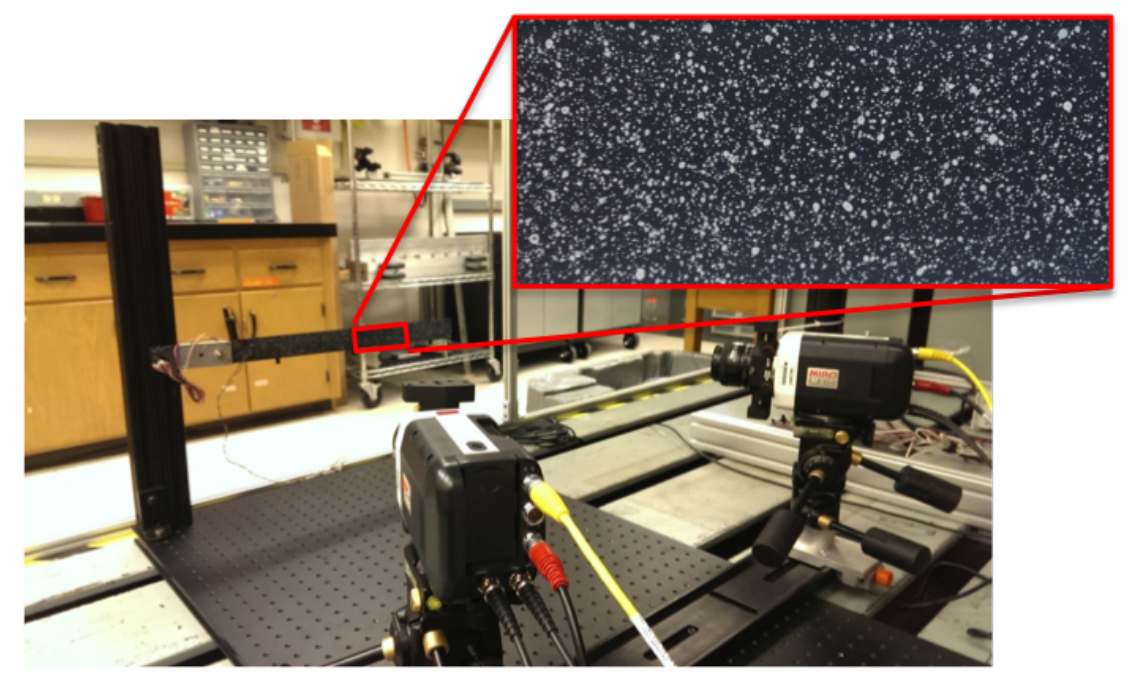

Figure 4.5: Cantilever beam with random speckle pattern. High-speed cameras can be seen in the foreground. Close-up of speckle pattern shown in image inset.

\subsubsection{Camera Settings and Lighting}

The camera lens aperture determines the depth of the field of focus. A smaller aperture increases the image depth of focus but requires more illumination. For this test, the f-stop, or ratio of focal length to aperture diameter, was selected to be 5.6. 
The exposure time of the camera sets how long the shutter will remain open for each image capture. A shorter exposure time decreases image blur of fast-moving objects, however more lighting is required. The exposure time for this test was set to $31 \mu \mathrm{s}$.

The image capture rate must be selected according to the expected frequencies of vibration. Similar to modal analysis in the frequency domain, the sampling frequency must be greater than twice the highest frequency of interest. However, sampling at too high a frequency decreases the ability to resolve vibration at lower frequencies. This is because the response matrices are formulated on the shift between three successive measurements, so if not enough modal displacement has occurred within that time frame, the ITD procedure cannot extract accurate modal parameters. Based on the analysis of numerous experiments, it was determined that the sampling rate must be less than 100 times the lowest frequency of interest. The sampling rate can be artificially halved by only analyzing every other image. Similarly, every third, fourth, etc. image can be analyzed to reduce the sampling rate further. For this test, images were captured at a rate of $1000 \mathrm{~Hz}$, although the ITD analysis was performed on every other image, making the effective sampling rate $500 \mathrm{~Hz}$.

The entire region of interest must be in focus for the DIC to produce accurate deformations. If the plane of the test article is not parallel to the plane of focus of the camera, a Scheimpflug adaptor can be used to adjust the angle of the lens relative to the sensor (image plane), which rotates the plane 
of focus.

Proper illumination is critical for image correlation. It is recommended that the average pixel intensity be above 80 counts[18]. Additionally, reflections from the light source that remain stationary as the test article moves (i.e. glare) can distort the produced deformation vectors. Finally, if possible, illumination should be provided by a flicker-free source such as sunlight or a flicker-free studio light . A pair of incandescent lamps were used to illuminate the cantilever beam in this test.

\subsubsection{Calibration}

The DIC calibration defines both a mapping function that transforms camera image coordinates into real world coordinates and a dewarping function to correct for image distortions. Calibration is performed by placing a calibration plate with markers of known size and spacing approximately at the location of the test article. By capturing images of the calibration plate and identifying markers in the images from both cameras, the DIC software can calculate a mapping function that is valid over the entire region of focus. The DIC software does not require the calibration plate to cover the entire field of view, and it only requires a single view of a 2D surface to generate an acceptable mapping function[18]. However, increasing the image area covered by the calibration plate and capturing multiple images of the plate at different heights and tilt angles can increase the accuracy of the mapping function. The software also calculates the positions of the cameras relative to the calibration 
plate and the average standard deviation in pixels to the marker locations. A good calibration will have a standard deviation of less than 0.3 pixels [18]. For this experiment, calibration was performed with a $310 \mathrm{~mm}$ by $310 \mathrm{~mm}$ calibration plate with targets imprinted on the surface and in grooves to provide a three dimensional array of reference points.

\subsubsection{Image Acquisition and Deformation Calculation}

A series of images must be captured of the test article undergoing vibration. The deformation will be calculated relative to some reference image, so an image pair of the stationary test article must be captured as well. For this test, 3000 images were captured starting with with the beam at rest. The beam was impacted with a small hammer near the midspan of the beam at image 98 , or $0.098 \mathrm{~s}$ after recording began. The last image before the hammer impact was chosen to be the reference image, and the previous images were discarded.

Once the 3D mapping and dewarping functions have been defined and the images of the vibrating test article have been captured, DIC can be performed to calculate the deformation of the test article in each image. The reference image from one camera is divided into a grid of interrogation windows. Each interrogation window possesses a unique intensity pattern defined by the speckles contained within the window. The DIC algorithm then identifies the same intensity pattern in the image from the other camera. The image coordinates of the patterns are passed through the mapping and dewarping 
functions to identify the spatial location of each group of speckles. The same intensity patterns are identified in each subsequent image pair, and the shift in pixels between the patterns in the reference image pair and the following image pairs is used to determine the 3D displacement of each group of speckles. Each interrogation window produces a single deformation vector.

The deformations in each image pair can be calculated from the pixel shift relative to the reference image or relative to the preceding image. When the expected deformations are small, it is preferable to calculate the deformation relative to the reference image. However, if the test article has moved a large distance relative to the reference image, the pixel shift may be too great to find correlation. In that case, the deformation can be calculated relative to the preceding image, and the deformations between each image can be summed to determine the deformation relative to the reference image. While this allows correlation to be maintained even over large deformations, the error from each calculation is propagated through the subsequent deformation calculations. As the deformations were small for this test, correlation was performed relative to the reference image.

The spatial resolution of the deformation vectors is determined by the size of the interrogation windows and the overlap between windows. Typical window sizes range from $16 \times 16$ to $64 \times 64$ pixels with $50 \%$ to $75 \%$ overlap between windows. Larger sized windows generally yield more accurate estimates of deformation, however fewer deformation vectors will be produced. Additionally, window sizes that are too large may not accurately determine the 
deformation of regions with large strain gradients. The interrogation window size for this test was selected to be 31 pixels with a $75 \%$ overlap between windows. This produced 1842 deformation vectors over the surface of the beam with a spatial resolution of $2.67 \mathrm{~mm}$ between vectors. Recall that for the experiment in Section 4.1, an ITD analysis was performed using the deformation at 80 locations. It is apparent that DIC produces more than sufficient numbers of deformation vectors to formulate an ITD analysis.

The DIC algorithm requires at least one location to begin the correlation mapping and serve as an initial guess for the surrounding deformation vectors. These seeding points should be located in an area visible to both cameras and where relatively small deformation is expected.

An image mask may be defined to exclude pixels outside the area of interest, decreasing the computation time. An example of a masked and seeded image is shown in Figure 4.6.

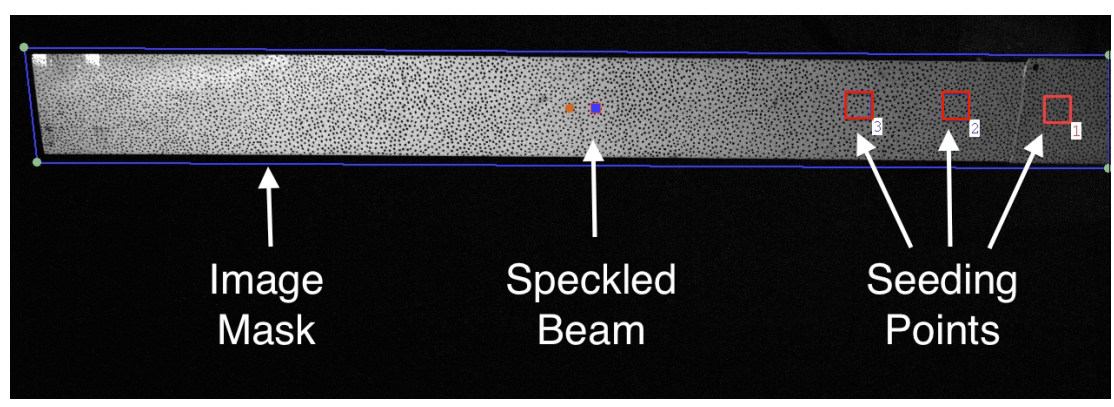

Figure 4.6: Example mask and seeding points.

Post-processing tools can be used to increase the accuracy of the calculated deformations. Applying an outlier filter will remove vectors that deviate 
from their neighbors by a specified amount. A smoothing filter will apply a 2nd order polynomial smoothing function to vectors calculated from a specified surrounding area size. The smoothing filter can also fill in missing vectors removed by the outlier filter. Both post-processing tools were used for this test.

The deformation measurements can be exported in text (ASCII) format. The three component deformations are exported with the $2 \mathrm{D}$ coordinates of the vectors. An example deformation field from a rap test is shown in Figure 4.7 .

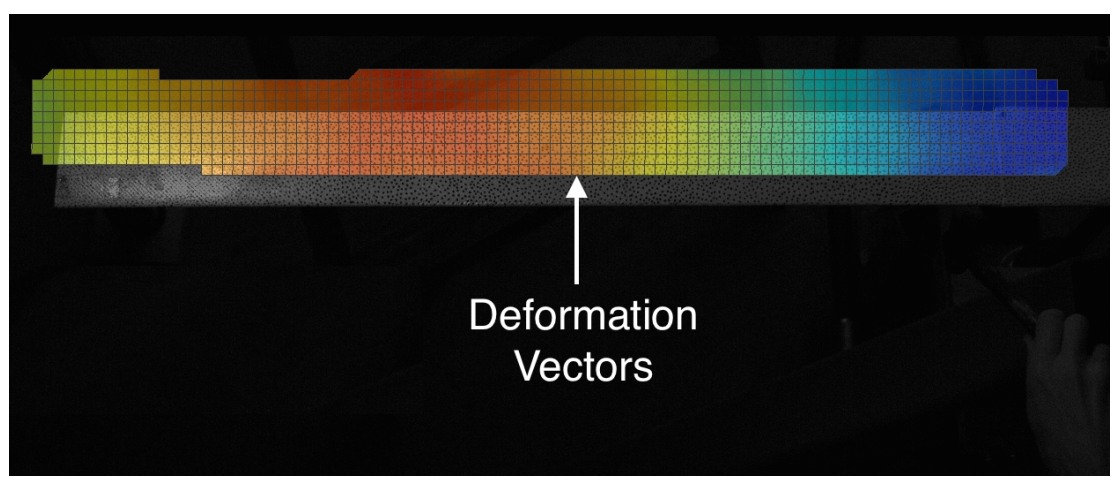

Figure 4.7: Example deformation field from rap test. Each box corresponds to an individual deformation vector. Red regions indicate larger deformations, and blue regions indicate smaller deformations.

\subsubsection{Formulation of the ITD Response Matrices}

A suite of MATLAB codes was developed to perform an ITD analysis based on the vibration data determined through DIC. Because DIC produces deformation vectors at far more locations than is required to formulate an 
ITD analysis, the user can select which locations will be used to assemble the response matrices. Additionally, the user may select what time instants will be used to assemble the response matrices. This includes the time shift between matrices $\Delta t$ in Eq. (3.4), the interval of time between measurements $y\left(x, t_{1}\right), y\left(x, t_{2}\right), \ldots$ in Eq. (3.3), and the ratio of time instants to measurement locations as described in Section 3.3. For this experiment, 440 locations along the span of the beam at 3 chord-wise positions were chosen to assemble the response matrices. The chosen locations overlaid on the available locations is shown in Figure 4.8.

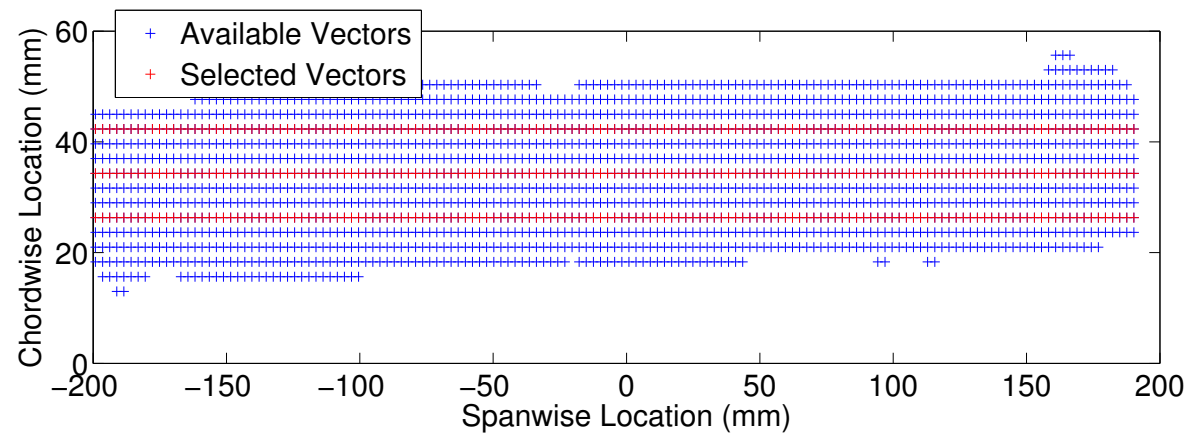

Figure 4.8: Selected deformation vectors for ITD analysis overlaid on the full range of deformation vectors calculated by DIC over the surface of the beam.

The response at these locations was measured at 883 time instants. The first 880 time instants were required to formulate the response matrix in Eq. (3.3). Two more time instants were required to formulate the matrices in Eq. (3.6) and Eq. (3.8). One last time instant was required to derive a new set of solutions to be compared to the first using the MSCC as defined in Eq. (3.24). The deformation along the center constant chord strip in Figure 4.8 at 
three successive time instants is shown in Figure 4.9.

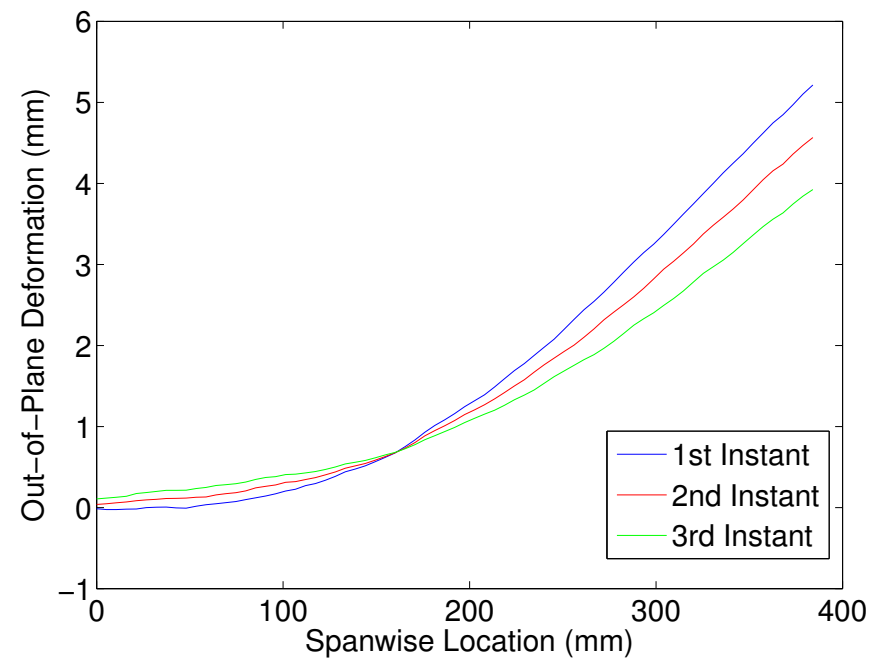

Figure 4.9: Sample response at selected analysis locations. Each time instant is separated by $0.001 \mathrm{~s}$.

Finally, thresholds for separating computational modes from structural modes must be set. Two ITD analyses are performed on time-shifted matrices in order generate two sets of modal parameters. Modes from each analysis that possess similar natural frequencies to within a specified threshold are then compared using the MSCC. If the MSCC has a value higher than a specified threshold, then the two modes are considered to be the same structural mode. Modes that do not pass the frequency matching and MSCC tests are assumed to be computational modes. For this test, the frequencies of two modes were required to be within $5 \%$ of one another and possess an MSCC greater than 0.999 in order to be classified as a structural mode. 


\subsubsection{Results of Cantilever Beam Experiment}

ITD analysis shows that the response was dominated by three modes. A Fast Fourier Transform (FFT) was performed on the response measured by DIC at one location at the tip of the beam, and an analytical solution was determined for an aluminum cantilever beam of the same dimensions using Euler-Bernoulli beam theory. A comparison of the natural frequencies determined by the three methods is shown in Table 4.2. The mode shapes determined by the ITD analysis and the Euler-Bernoulli method are compared in Figure 4.10, and the results of the FFT are shown in Figure 4.11.

\begin{tabular}{|c|c|c|c|}
\hline Mode & $\begin{array}{c}\text { ITD Frequency } \\
(\mathrm{Hz})\end{array}$ & $\begin{array}{c}\text { FFT Frequency } \\
(\mathrm{Hz})\end{array}$ & $\begin{array}{c}\text { Analytical Frequency } \\
(\mathrm{Hz})\end{array}$ \\
\hline First Flap & 3.08 & 3.00 & 2.82 \\
\hline Second Flap & 16.18 & 16.00 & 17.65 \\
\hline Third Flap & 43.97 & 43.99 & 49.41 \\
\hline
\end{tabular}

Table 4.2: Cantilever beam natural frequency comparison from ITD, FFT, and analytical analyses. 
The natural frequencies from the three solutions show good agreement. In particular, the natural frequencies from the ITD and FFT solutions are very close, differing at most by $2.5 \%$. The analytical solution differs slightly from the other two solutions because the test article is not an ideal beam and the root was not perfectly clamped. The mode shapes produced by ITD analysis follow the shapes predicted by beam theory, although there is some deviation. Again, this can be attributed to the difference between the assumptions of the Euler-Bernoulli theory and the actual conditions of the test. The damping ratios determined from this test are listed in Appendix A. 


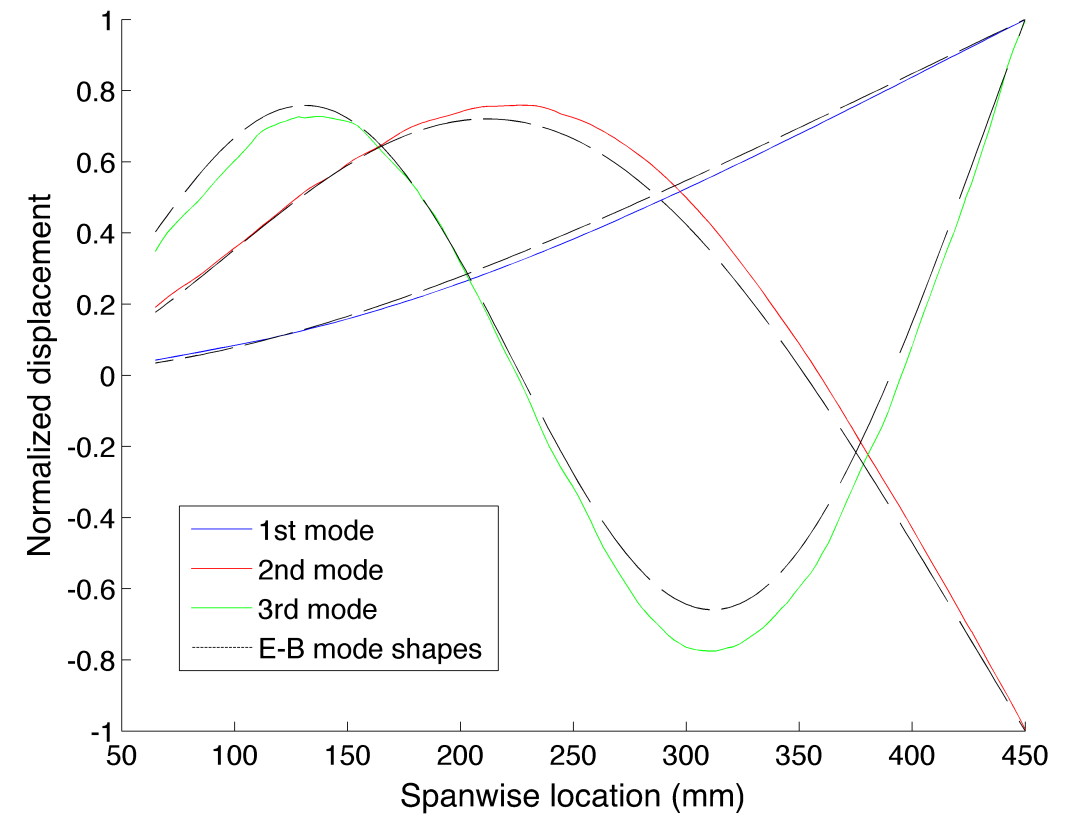

Figure 4.10: Comparison of ITD and Euler-Bernoulli mode shapes. The ITD mode shapes agree with the analytical predictions, although there is some difference as the actual beam was not perfectly cantilevered. 


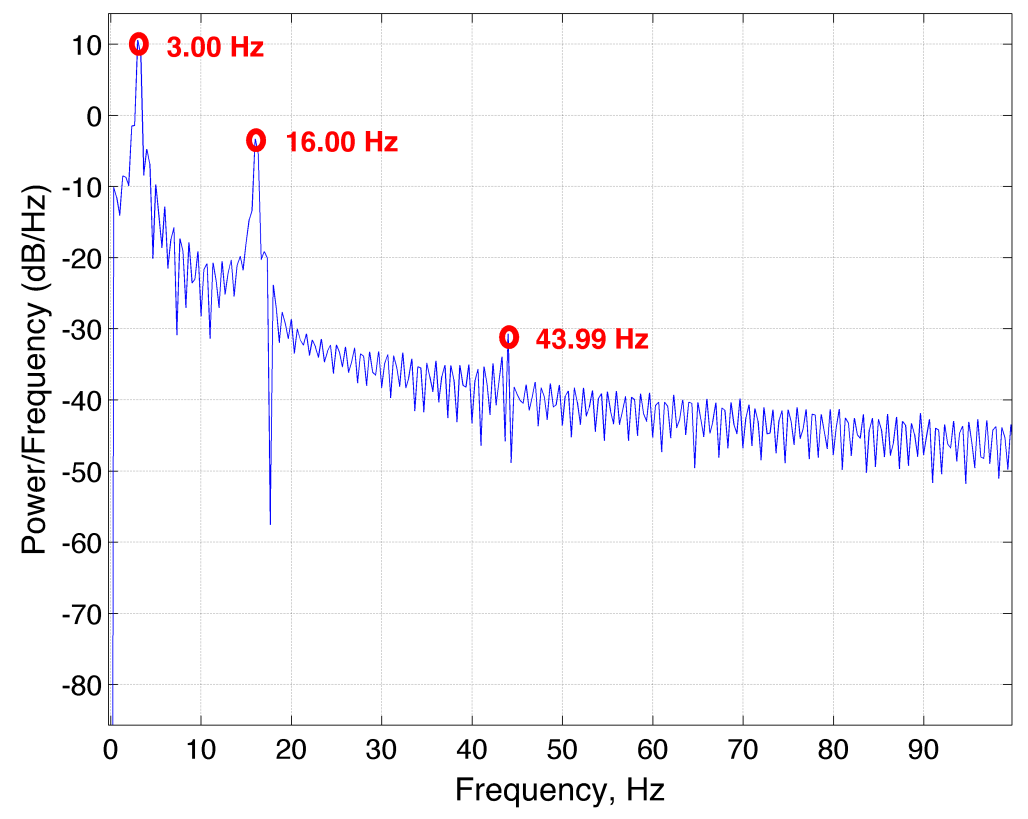

Figure 4.11: FFT of the deformation measured by DIC at the tip of the cantilever beam. The peaks circled in red correspond to the first three flap bending modes. 


\subsection{Accuracy of DIC Measurements and Modal Param- eters}

The nominal accuracy of deformation vectors calculated by DIC is solely a function of window size, with larger windows being more accurate. According to data provided by the DIC software vendor (LaVision[18]), a window size of $13 \times 13$ pixels will have an accuracy of 0.013 pixels, and a window size of $31 \times 31$ pixels will have an accuracy of 0.005 pixels[18]. However, this assumes the deformation was calculated using high-quality pictures, an ideally speckled test article, and a perfect calibration. Studies have shown that errors introduced in the calibration procedure [19] and through the interpolation of pixel intensity due to low-contrast speckles [20] are non-negligible. Sicard and Sirohi[13] concluded that including these additional sources of error causes the accuracy to be on the order of 0.01 pixels for in-plane displacements and 0.1 pixels for out-of-plane displacements. This will be the assumed accuracy for this study.

It is important to note that the accuracy of the measured deformation does not directly impact the accuracy of the calculated modal parameters, which are determined from relative motion of different locations on the test article. Thus, any bias in the measured deformation will not impact the mode shapes. Also, it is difficult to derive uncertainty in the modal parameters from the precision uncertainty in the measured deformations due to the noisecanceling effect of computational modes as described in Section 3.2 and the double least squares approximation as described in Section 3.3. The best 
approximation of the error of calculated modal parameters is determined from the scatter from multiple analyses.

One weakness of the ITD procedure is in calculating damping factors. Ibrahim [17] notes that due to the exponential nature of Eq. (3.16), a small error in the eigenvalues $\alpha_{r}$ will result in a large error in the calculated damping ratio in Eq. (3.21). The damping ratio error $\delta$ as a function of the eigenvalue error $\epsilon$ is calculated as,

$$
\delta=\frac{100}{\pi f \zeta_{\text {act }} \Delta t} \ln (1+\epsilon / 100)
$$

where $f$ is the natural frequency, $\zeta_{a c t}$ is the actual damping ratio, and $\Delta t$ is the time shift between matrices in Eq. (3.4). For example, if the error in the magnitude of a calculated eigenvalue is $2 \%$, the natural frequency is $20 \mathrm{~Hz}$, the actual damping ratio is 0.02 , and the sampling frequency is $500 \mathrm{~Hz}$, the error of the damping ratio would be approximately $790 \%$. Implementation

of the double least squared approximation reduces the error in the damping ratios, but the error is still much larger than that for the natural frequencies and mode shapes. The damping ratios will therefore not be discussed in this study.

\subsection{Conclusions of Preliminary Experiments}

The results of these experiments show not only that ITD analysis can accurately determine the modal parameters of a vibrating cantilever beam, but also that high-speed DIC can measure the deformation used by ITD analysis. 
In the following chapter, this procedure is extended to measure the modal parameters of a rotating helicopter blade. 


\section{Chapter 5}

\section{Helicopter Blade Experiment}

An experiment was performed to determine the modal parameters of a $2.032 \mathrm{~m}$ diameter composite helicopter rotor blade at rotational speeds up to 900 RPM. The goals of this experiment are to demonstrate the procedure to extract modal information from rotating cantilever beams using ITD and DIC, determine the accuracy of the measured deformations and extracted modal parameters, and investigate how the modal parameters of the rotor blade change with increasing rotational speed.

In this chapter, the experimental setup is described along with the procedure to capture the deformation data using DIC and formulate an ITD analysis of a rotating beam. This differs from a non-rotating test in that the images captured of the beam are not in a continuous series. Instead, images are captured at fixed azimuthal positions for every revolution of the beam. A Hall Effect sensor detects the passage of a magnet fixed to the shaft of the

rotor, which triggers image acquisition. Thus, for a fixed rotational speed and image acquisition rate, images will be captured of the beam at the same azimuthal position. 


\subsection{Geometry of Test Setup}

A single helicopter rotor blade with a counterweight was mounted to a UT designed and built rigid rotor stand, which was designed to be a stable platform for tests of large diameter rotors. A detailed description of the rotor blade is found in Section 5.2. The rotor was spun by a $100 \mathrm{hp} \mathrm{hydraulic} \mathrm{motor.}$

Cameras and light fixtures were mounted to a $4.6 \mathrm{~m}$ tall frame assembled from 80/20 aluminum extrusions, which was also designed and built in-house. A camera rail was mounted on extendable booms, which allowed the cameras to be placed directly over the rotor disk. The experimental setup is shown in Figure 5.1.

Images were captured using a pair of Vision Research Phantom Miro M310 high-speed digital cameras. Each camera is capable of recording 3200 images per second at full $1280 \times 800$ pixel resolution. The $25.6 \times 16.0 \mathrm{~mm}$ CMOS camera sensor has a 12-bit resolution, and the minimum exposure time is $1 \mu \mathrm{s}$. The cameras were used with Nikon AF Nikkor 35mm f/2D lenses, an f-stop of 5.6, and LaVision Scheimpflug adaptors. Images were processed using the LaVision DaVis 8.2.2 software package, which both controlled the image capture and performed Digital Image Correlation on the saved images.

A LaVision High-Speed Controller synchronized image capture with an external once-per-revolution trigger provided by a Hall Effect sensor, which was also used to monitor rotor speed. A single-axis PCB Integrated Circuit Piezoelectric accelerometer was used to monitor the vibration of the test stand 


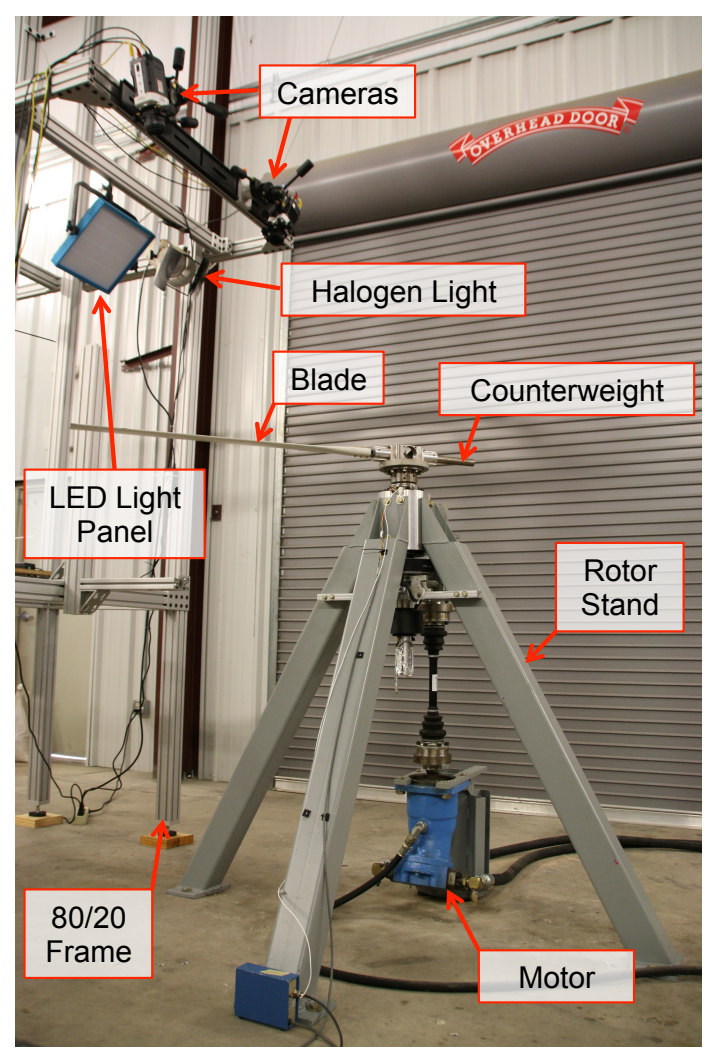

Figure 5.1: Helicopter blade experiment setup. Cameras and light fixtures are mounted to the 80/20 frame. Image capture, hydraulic motor control, and sensor monitoring is managed from inside a control room.

during operation. Signals from the two sensors were acquired with a National Instruments PXI-6358 DAQ and LabVIEW. The Hall Effect sensor and accelerometer are shown in Figure 5.2. 


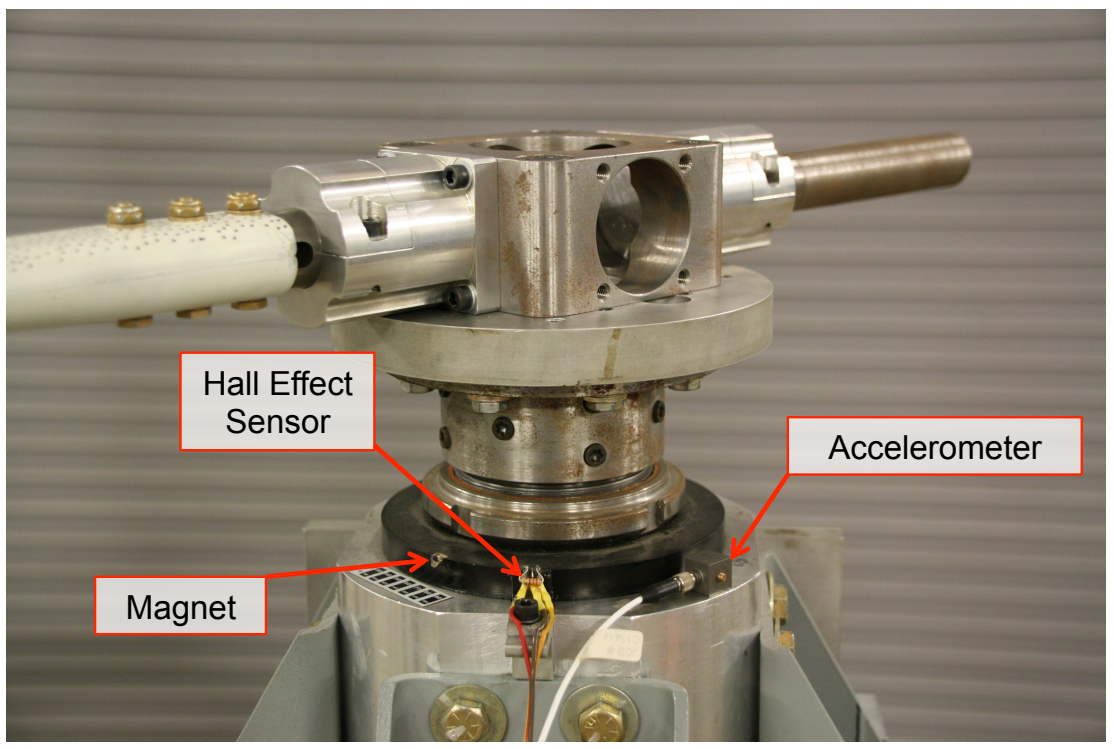

Figure 5.2: Hall Effect sensor and piezoelectric accelerometer mounted to rotor stand. The magnet is mounted to the rotating shaft.

The rotor blade was excited by a compressed air nozzle oriented normal to the rotor disk that shot a constant jet of air through the plane of the rotor. The span-wise location of the air nozzle and the distance to the rotor disk was varied between tests to excite different structural modes. The hose pressure of the compressed air was approximately 90 psi.

The geometric arrangement of the cameras, rotor blade, and air nozzle is shown in Figure 5.3 and Figure 5.4. 


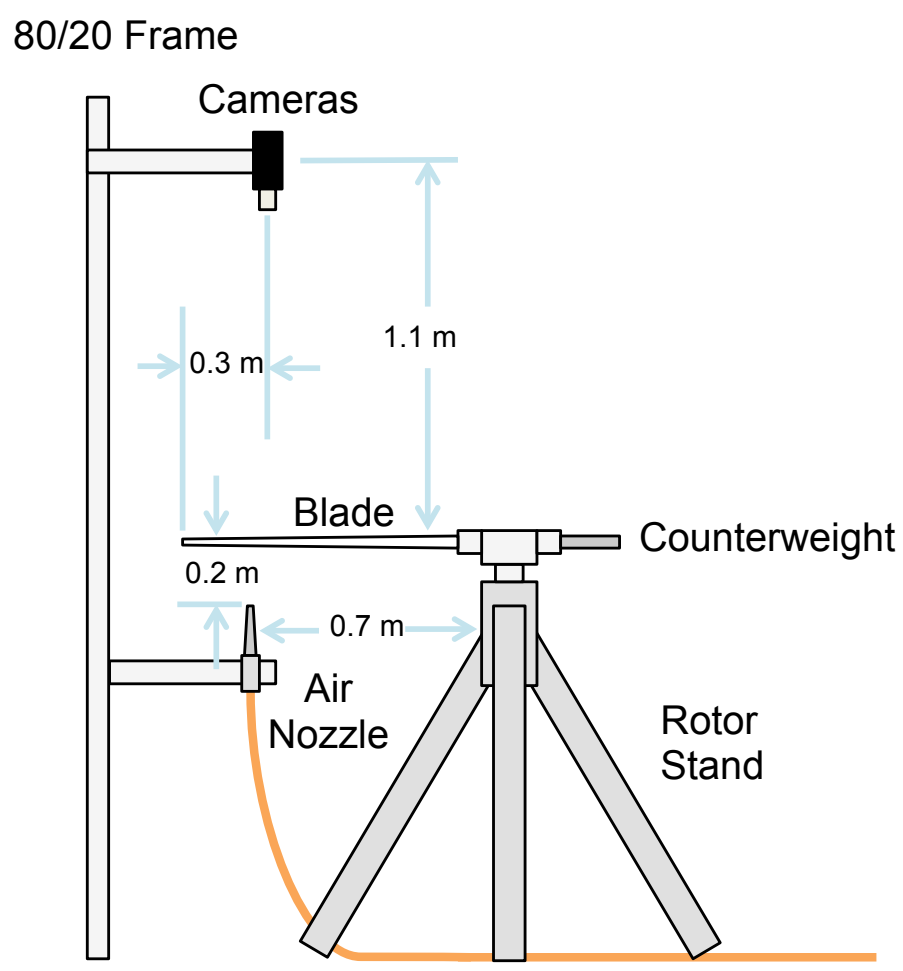

Figure 5.3: Schematic of test setup, side view. An example placement of the air nozzle is shown. 


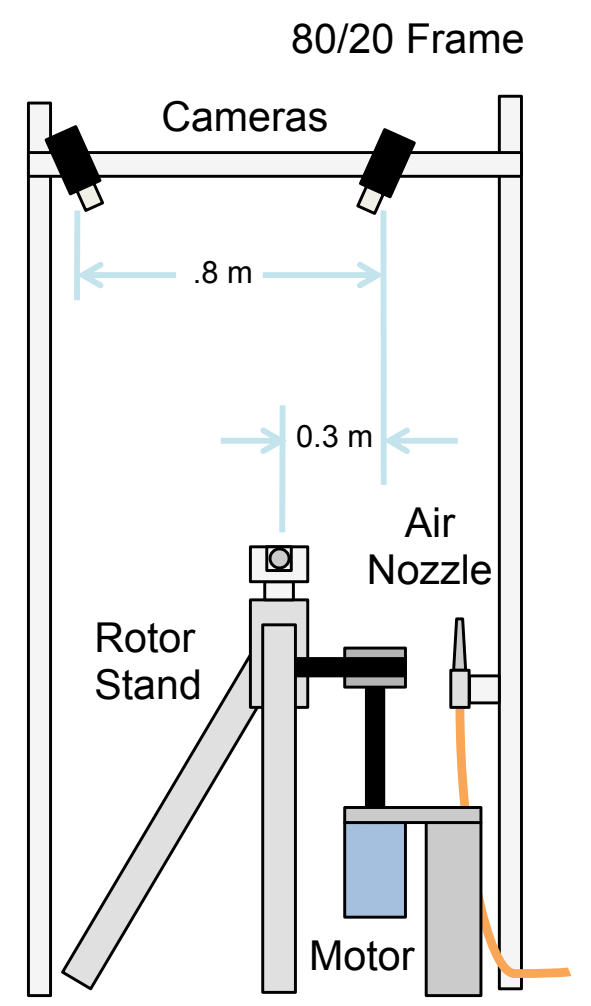

Figure 5.4: Schematic of test setup, front view. An example placement of the air nozzle is shown.

Note that the cameras are not centered on the rotor stand. The cameras were positioned so that the first image of every revolution would be aligned horizontally at the edge of the field of view. The subsequent images would show the rotor blade sweeping through the rest of the field of view. This is because the DIC algorithm calculates the deformation at locations defined in the first image, so the resulting grid in this case will be oriented approximately horizontally. This simplifies selecting locations for ITD analysis. The spacing of the imaged azimuthal positions is determined by the rotational speed and 
the image acquisition rate, and the number of images that can be captured per revolution is determined by the field of view. A visualization of the image capture sequence is shown in Figure 5.5.

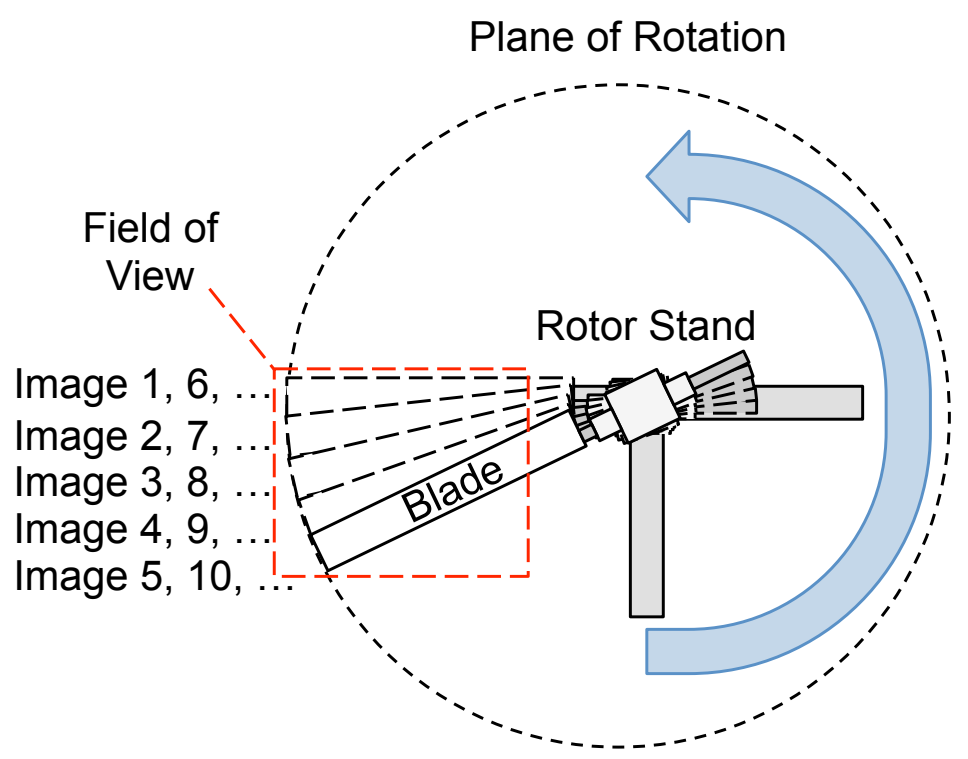

Figure 5.5: Imaging sequence of blade passage. At a rotational speed of 900 RPM, five images of the blade may be captured at a rate of $1000 \mathrm{~Hz}$ before the blade moves out of the field of view.

\subsection{Test Article}

The test article under investigation is a single blade of a $2.032 \mathrm{~m}$ diameter, Mach-scale helicopter rotor[21]. The composite blade is composed of a foam core wrapped with carbon fiber cloth, with an additional carbon-fiber stiffening cuff near the root. The blade profile and cross sections are shown in Figure 5.6. 


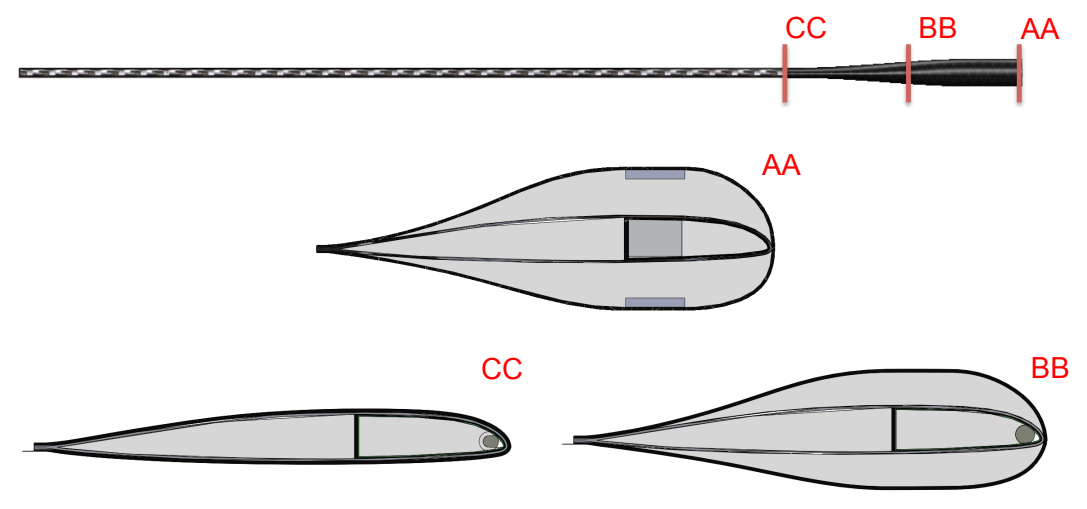

Figure 5.6: Blade profile and cross sections. The blade has a VR-12 airfoil section, a chord length of $0.08 \mathrm{~m}$, and a design tip speed of $190 \mathrm{~m} / \mathrm{s}$.

The blade is mounted to a blade grip, which is in turn rigidly mounted to a rotor hub. The pitch angle of the blade is set at approximately 0 degrees to minimize rotor thrust and resultant upward bending of the rotor blade. A steel counterweight is mounted to the opposite side of the rotor hub with another mounting grip.

A stochastic pattern of black speckles on a white matte background was applied to the blade. The speckles were sized to be approximately $1 \mathrm{~mm}$ in diameter with 1 - 3 mm spacing between the speckles. With a $35 \mathrm{~mm}$ lens at a distance of $1.1 \mathrm{~m}$, this corresponded to a speckle diameter of 2 - 3 pixels. A picture of the speckled test article is shown in Figure 5.7. 


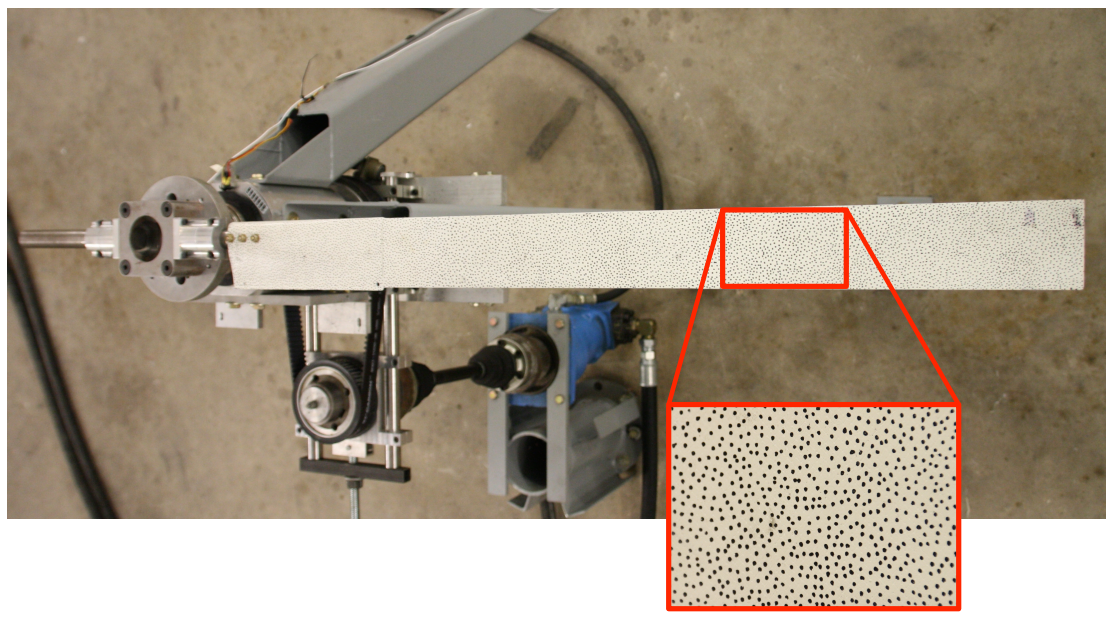

Figure 5.7: Top view of rotor blade mounted to rotor stand. Close up view of speckle pattern shown in image inset.

\subsection{Camera Settings and Lighting}

The field of view of the cameras on the rotor plane was approximately $50 \times 75 \mathrm{~cm}$, which corresponded to a maximum azimuthal range of 30 degrees The outer $70 \mathrm{~cm}$ of the blade were contained in this field of view, which includes the outermost portion of the stiffening cuff. From previous experiments, it was determined that the bending deformation of the stiffening cuff was negligible compared to the deformation of the outboard section of the wing when the blade was vibrating. Thus, the stiffening cuff was excluded from the field of view in order to improve the spatial resolution on the rest of the blade.

A series of images was captured at a rate of $1000 \mathrm{~Hz}$ every time the Hall Effect Sensor detected the passage of the magnet attached to the rotor shaft. At the maximum rotational speed of 900 RPM, this allowed for 5 image 
captures per revolution before the rotor blade would move out of the field of view. The aperture of the camera lens was set to $5.6 \mathrm{f} / \mathrm{D}$.

The exposure time was selected based on the maximum allowable blur at the tip of the blade. From the results of previous experiments, this was determined to be $1 \mathrm{~mm}$. At $900 \mathrm{RPM}$, this corresponded to an exposure time of $11 \mu \mathrm{s}$.

The test article was illuminated by a Dracast LED1000 Pro Daylight light panel, which provided flicker-free illumination. When very short exposure times were required, additional lighting was provided by a Smith Victor Q60 halogen light.

\subsection{Calibration}

Before each test, a calibration was performed to determine the mapping function to convert image coordinates into physical coordinates. A Type 1000 calibration plate $(0.8 \times 1 \mathrm{~m})$ was mounted in the field of focus of the cameras, and a series of three images of the plate were averaged to produce the calibration image. The resultant standard error of fit varied between 0.1 and 0.15 pixels between calibrations. The calibration plate mounted in place is shown in Figure 5.8. 


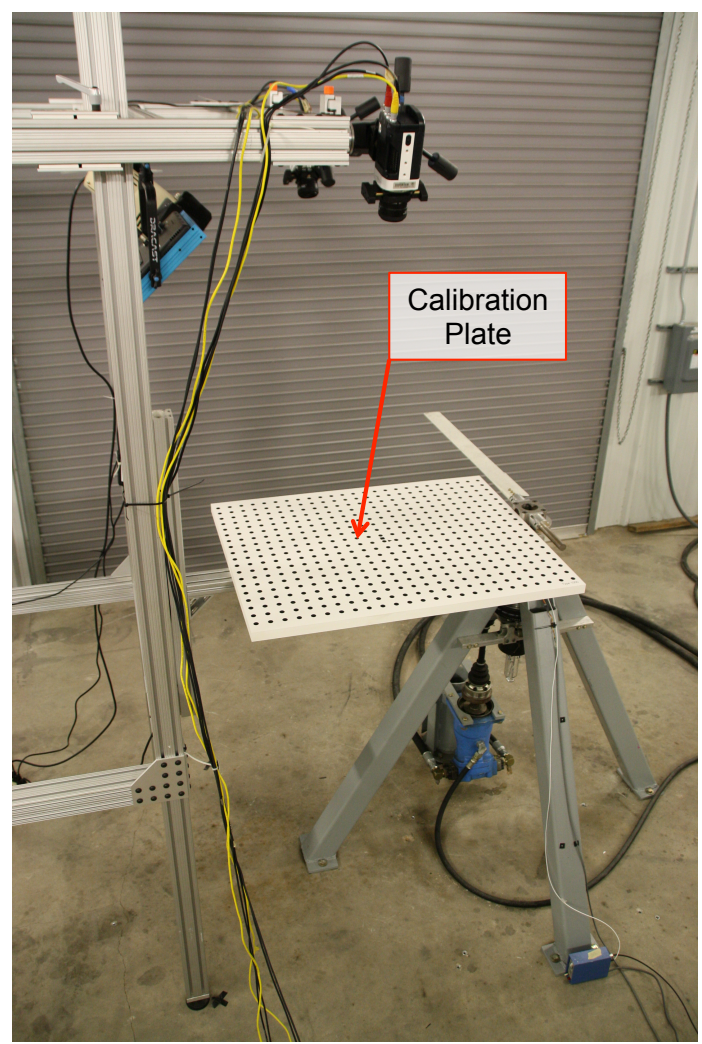

Figure 5.8: Calibration setup. Calibration plate is mounted to rotor hub and $80 / 20$ frame. The plate is large enough to cover the field of view of the cameras.

\subsection{Image Acquisition and Deformation Calculation}

For the non-rotating test, the blade was struck with a small hammer and 1200 images were captured of the blade as it vibrated. The impact occurred at the 110th image, or 0.11 seconds after recording began.

For rotating tests, 100 unexcited blade passage were recorded, and 500 excited blade passage were recorded. The unexcited blade passages are recorded to determine the rigid body motion and elastic deformation of the 
blade that is not caused by the applied excitation. The unexcited deformations will be averaged and subtracted from the excited blade passages, leaving only the deformation from the vibration of the blade around the baseline deformation. A set of 500 images of the stationary blade positioned at the same azimuthal position as the first image of each revolution was also captured to provide a reference for DIC calculation.

\subsection{DIC Settings}

The subset size (interrogation window size) for performing the DIC was set to 31 pixels. The step size was chosen to be 12 pixels, which corresponds to a $61 \%$ overlap between windows. These settings produced a vector grid with a spatial resolution of 12 pixels between vectors. With the geometric arrangement shown in Section 5.1, this corresponded to a physical spatial resolution of $7.2 \mathrm{~mm}$. A geometric mask was applied to the images to exclude regions away from the test article from the DIC calculations and decrease computation time. Three seeding points near the root of the test article were chosen as initial start points for the DIC algorithm. Approximately 850 vectors were calculated over the surface of the test article, though the total number of vectors varied slightly between tests due to small changes in setup and calibration.

The nominal accuracy of the DIC measurements for these settings according to the LaVision manual is $3 \mu \mathrm{m}$. The accuracy according to the study performed by Sicard and Sirohi[13] is $6 \mu \mathrm{m}$ for in-plane deformation and 60 
$\mu \mathrm{m}$ for out-of plane deformation.

For the non-rotating rap test, DIC was performed on images relative to an image of the blade before it was impacted by the hammer. For the rotating tests, an image of the stationary blade was inserted before the first image of each revolution. The deformation of the blade was then calculated relative to the image of the stationary blade. Because the blade moved through the entire field of view for each rotation, the pixel shift from the last images relative to the stationary image was too large to find correlation. Therefore, the deformation in each image after the first was calculated relative to the preceding image, and the total deformation was calculated as the sum of the deformations from all the previous images. This procedure was performed for both the unexcited blade passages and the excited blade passages.

\subsection{ITD Analysis Parameters}

ITD analysis was performed using deformation vectors at constant chord-wise locations along the blade. In order to generate a set of modal parameters that could be statistically analyzed, multiple ITD analyses were performed on strips of vectors at different chord locations. Additionally, the number of locations along each strip, the starting azimuthal position, and the ratio of time instants to measurement locations were varied. The sampling rate was also artificially reduced to $500 \mathrm{~Hz}$ by formulating the ITD analysis using the deformation from every second image.

Computational modes were separated from structural modes by requir- 
ing modes from two different formulations to have an MSCC greater than 0.95 and frequencies matching to within 5\%. These settings frequently did not filter

out all computational modes, forcing modes, and harmonics, so the expected frequencies and mode shapes were used to assess whether a produced mode was indeed a structural mode.

The accuracy of the modal parameters was determined from a $95 \%$ confidence interval of the mean of the collected parameters.

\subsection{Assumptions and Approximations}

The procedure outlined above makes certain assumptions and approximations in determining the modal parameters of rotating cantilever beams; these are described in this section. Suggestions to improve the current methodology and minimize or eliminate these assumptions are listed in Section 7.2.

One assumption is that the response due to excitation from the rotor hub is negligible compared to the response from the applied excitation. The applied excitation is an impulse before the beam enters the field of view of the cameras, so the measured response is approximated to be a free response as required by the basic ITD formulation in Section 3.1. If the excitation from the rotor hub were not negligible, then the response could not be approximated as a free response.

A second assumption is that because the applied forcing is periodic, the ITD analysis will produce a "forced mode" that can be identified and excluded. 
This forced mode will match the frequency of rotation and have a damping ratio close to zero.

A third assumption is that any identified mode with a natural frequency that is an integer multiple of another mode or the frequency of rotation is a harmonic and can be excluded. It is important to perform a non-rotating test to identify the expected natural frequencies so that structural modes are not accidentally assumed to be forced or harmonic modes. 


\section{Chapter 6}

\section{Results and Analysis}

\subsection{DIC Deformations}

The deformation of the blade was calculated at approximately 850 locations over the surface of the blade while rotating at 0, 300, 600, and 900 RPM. The deformation from 100 rotations with no applied excitation was averaged at each captured azimuthal position to determine the elastic deformation and rigid body motion of the blade out of the plane-of-rotation. This baseline deformation was subtracted from 500 excited blade passages to determine the bending vibration of the blade. The average baseline deformation and associated uncertainty is plotted with a sample excited deformation at 600 RPM in Figure 6.1. Three successive time instants separated by $0.001 \mathrm{~s}$ are shown, which corresponds to three different azimuthal positions. 


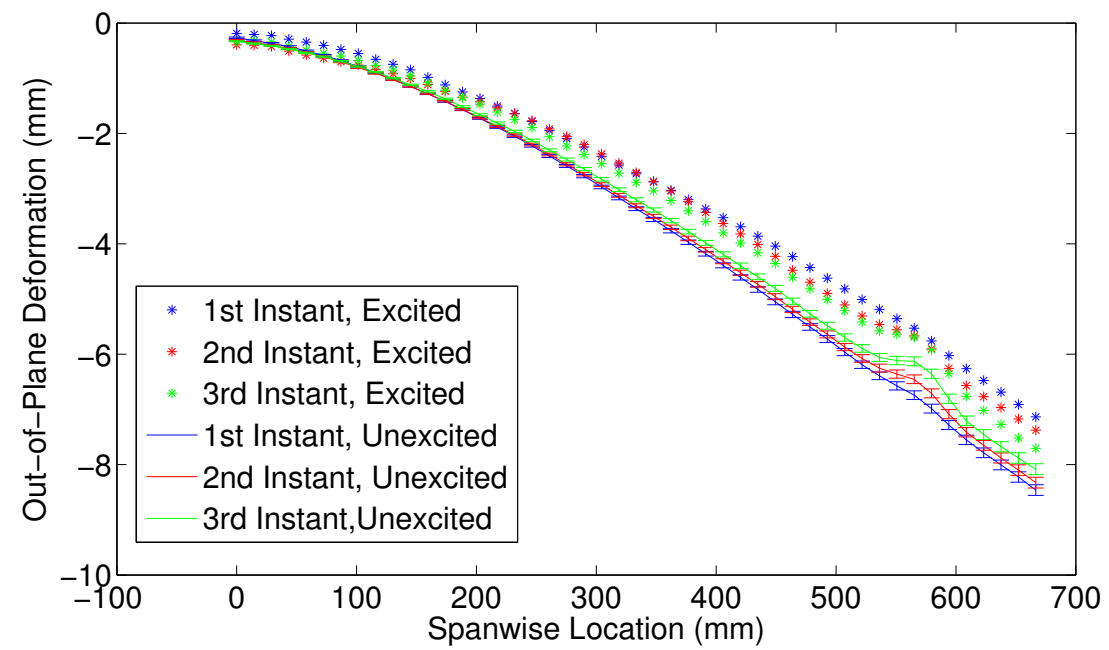

Figure 6.1: Excited and unexcited blade deformations at 600 RPM.

The magnitude of the baseline deformation is much larger than the vibration, which is shown by the proximity of the excited and unexcited deformation shapes. The variance of the baseline deformation is small, with $5 \%$ uncertainty at the root and $1 \%$ uncertainty at the tip. The negative deformation values indicate that the blade was positioned with a slight negative pitch angle, although the resultant elastic deformation at the tip is less than $1 \mathrm{~cm}$. The difference between the three time instants for the baseline measurements corresponds to the rigid body motion that arises due to the misalignment of the calibration plate with the plane of rotation.

There is some unusual deformation near the tip of the blade for both the excited and unexcited blade passages. Upon further investigation, it was concluded that this was caused by a region of glare on the blade. Because the glare remains stationary while the blade moves, the deformation calculated in 
this region differs from surrounding areas. This condition exists for both the excited and unexcited blade passages, so the bias is removed with the elastic deformation and rigid body motion.

The deformation of the blade with the elastic deformation and rigid body motion removed is shown in Figure 6.2.

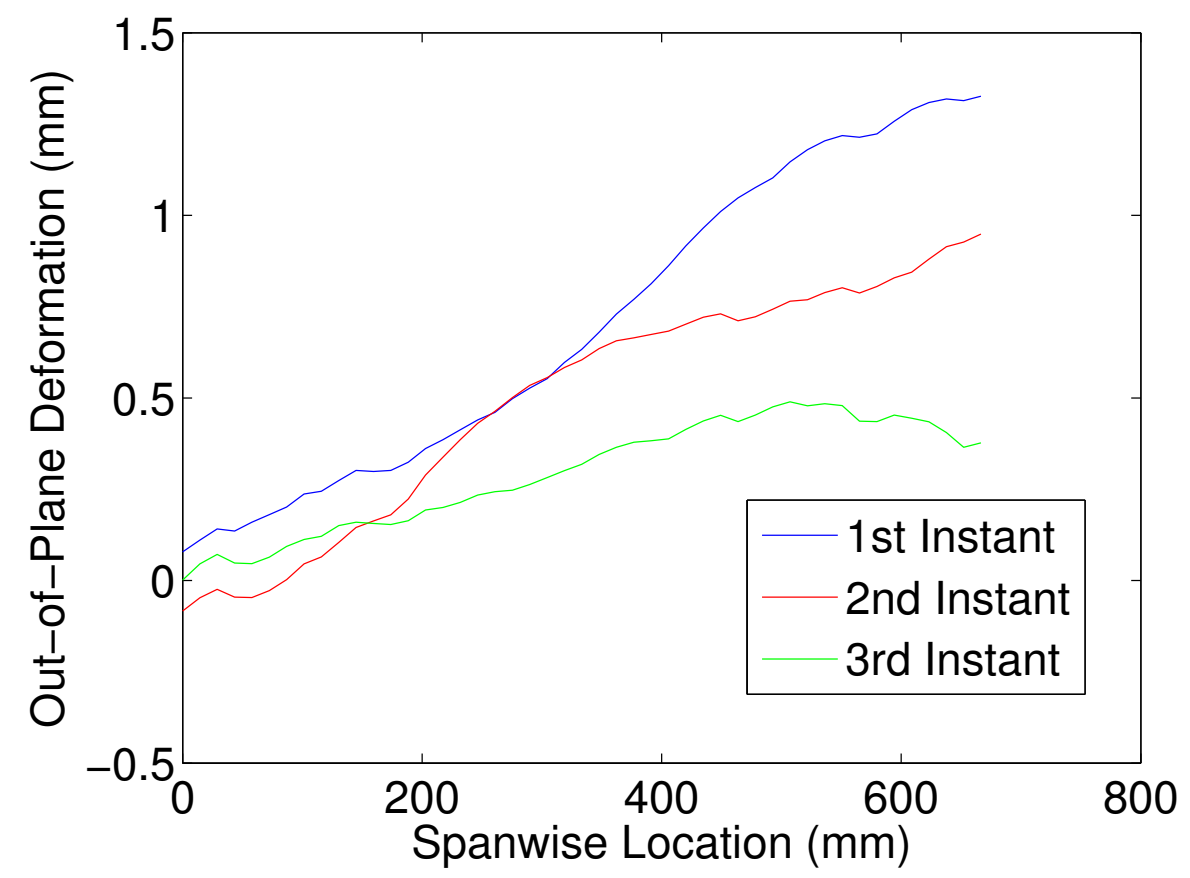

Figure 6.2: Excited blade deformations with rigid body correction at 600 RPM.

The series of corrected deformations indicate that the blade is flapping downward as it passes through the field of view. There is some random variation in the corrected deformation that is on the order of $50 \mu \mathrm{m}$. This is approximately the magnitude of the predicted uncertainty for out-of-plane deformation $(60 \mu \mathrm{m})$. 


\subsection{ITD Analysis Results}

The natural frequencies and mode shapes associated with the first three flap bending modes of the blade were determined from the corrected deformations. The natural frequencies and mode shapes are compared to an analytical model of the same rotor blade, developed by Schmaus and Chopra[22] using the University of Maryland Advanced Rotorcraft Code (UMARC). The analytical solution was developed by modeling the blade as a second-order, nonlinear, isotropic Euler-Bernoulli beam with flap, lag, torsion, and axial motion. A fan plot comparing the analytical and experimental natural frequencies at increasing rotational speeds is shown in Figure 6.3. The frequencies in the fan plot are normalized by 1800 RPM, which is the design speed of the blade. The natural frequencies are summarized in Table 6.1.

\begin{tabular}{|c|c|c|c|c|c|}
\hline Mode & & $0 \mathrm{RPM}$ & $300 \mathrm{RPM}$ & $600 \mathrm{RPM}$ & $900 \mathrm{RPM}$ \\
\hline \multirow{3}{*}{$\begin{array}{l}\text { First } \\
\text { Flap }\end{array}$} & Analytical (Hz) & 16.6 & 17.6 & 19.0 & 25.5 \\
\hline & Experimental (Hz) & $13.6 \pm 0.01$ & $14.5 \pm 1.2$ & $14.9 \pm 0.8$ & $18.8 \pm 1.6$ \\
\hline & Percent Difference & $22 \%$ & $21 \%$ & $28 \%$ & $36 \%$ \\
\hline & & & & & \\
\hline \multirow{3}{*}{$\begin{array}{c}\text { Second } \\
\text { Flap }\end{array}$} & Analytical (Hz) & 94.7 & 95.6 & 97.7 & 103.8 \\
\hline & Experimental $(\mathrm{Hz})$ & $81.9 \pm 0.01$ & $82.6 \pm 0.8$ & $83.1 \pm 0.6$ & $88.0 \pm 1.9$ \\
\hline & Percent Difference & $16 \%$ & $16 \%$ & $18 \%$ & $18 \%$ \\
\hline & & & & & \\
\hline \multirow{3}{*}{$\begin{array}{l}\text { Third } \\
\text { Flap }\end{array}$} & Analytical (Hz) & 245.2 & 246.1 & 249.0 & 254.7 \\
\hline & Experimental $(\mathrm{Hz})$ & $214.8 \pm 0.3$ & $195.5 \pm 12.7$ & $199.8 \pm 2.0$ & $209.7 \pm 1.9$ \\
\hline & Percent Difference & $14 \%$ & $26 \%$ & $25 \%$ & $21 \%$ \\
\hline
\end{tabular}

Table 6.1: Analytical vs. experimental natural frequencies. 


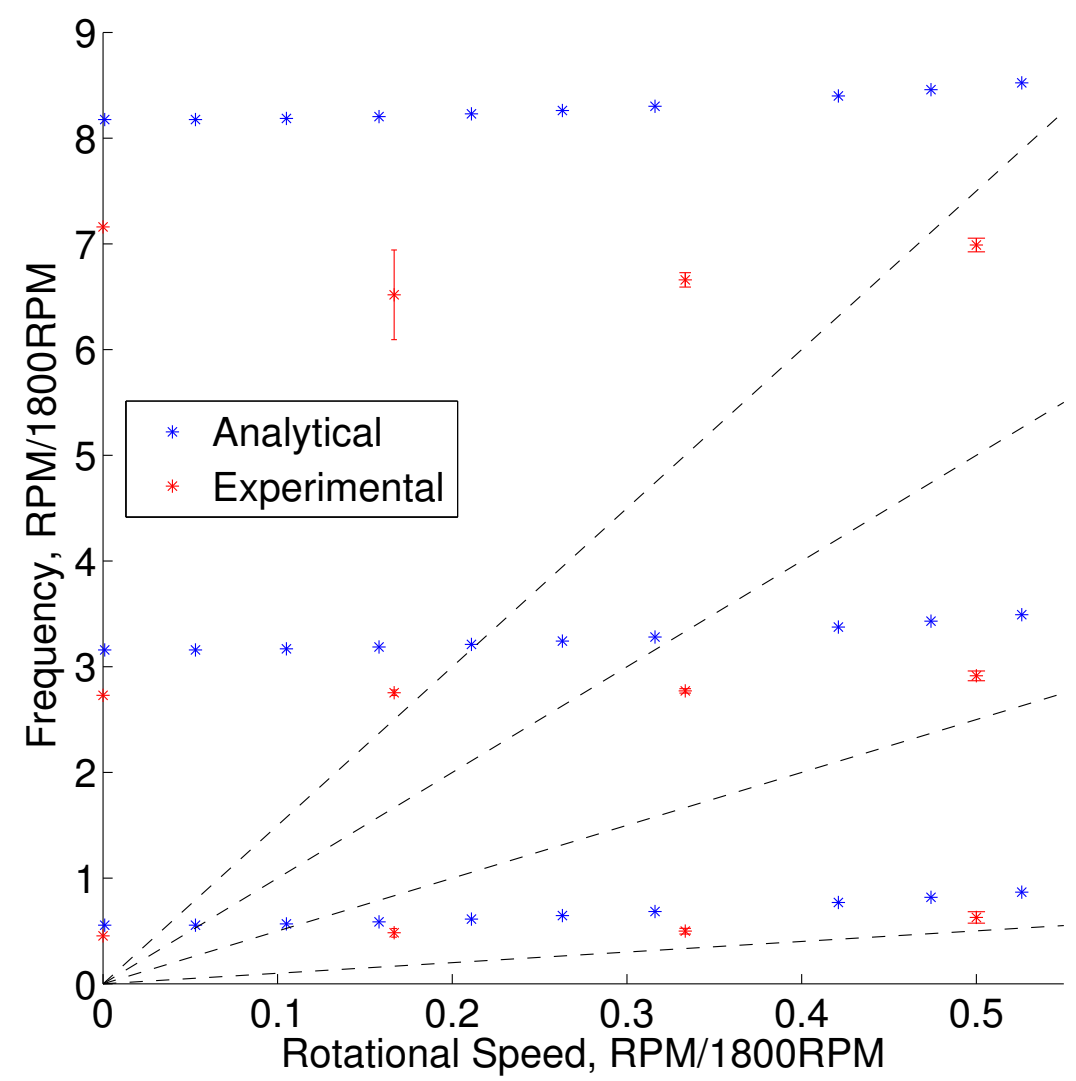

Figure 6.3: Natural frequencies vs. rotational speed. Dashed lines correspond to one, five, ten, and fifteen per revolution frequencies. 
The experimental natural frequencies are consistently lower than the analytical natural frequencies. In order to verify the results of the non-rotating test, a rap test was performed on the blade mounted in the rotor hub using an ICP accelerometer. The accelerometer was attached to the stiffening cuff of the blade, and the rotor hub was impacted with a small hammer. The vibration response was collected at a sampling rate of $1000 \mathrm{~Hz}$ over an interval of $1 \mathrm{~s}$, and an FFT was performed on the signal to determine the frequency content. The power spectrum averaged over five tests is presented in Figure 6.4 .

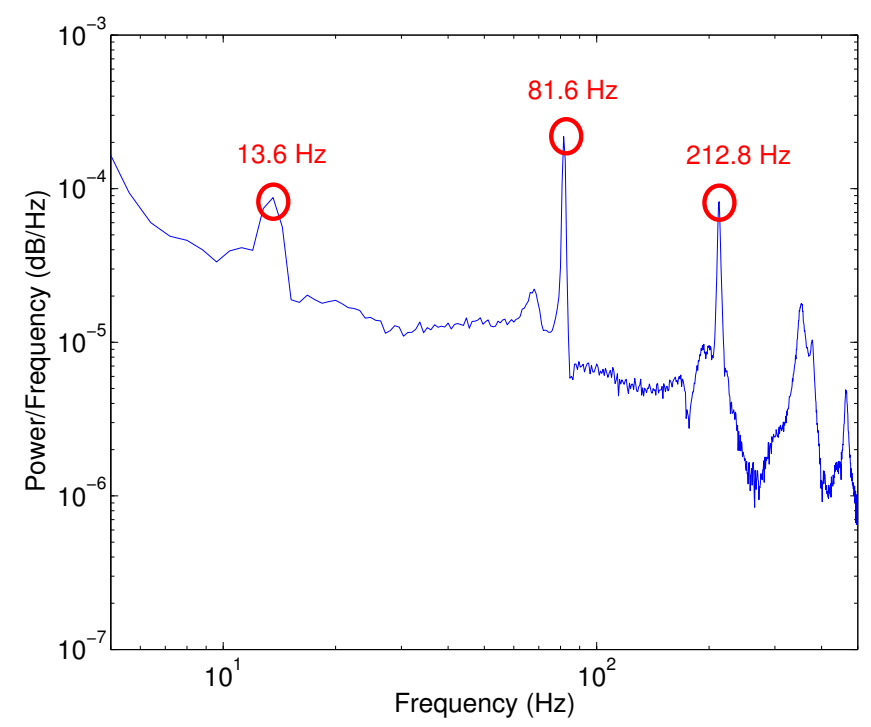

Figure 6.4: Averaged power spectrum of accelerometer response from rap tests. The first three flap bending natural frequencies are circled in red.

The peaks circled in red at 13.6, 81.6, and $212.8 \mathrm{~Hz}$ correspond closely with the first three flap bending natural frequencies determined from the ITD 
analysis at 0 RPM. This indicates that these are indeed the natural frequencies and that the UMARC simulation did not accurately predict the natural frequencies for these test conditions. The discrepancy is caused by differing boundary conditions between the simulation and the test. The UMARC simulation assumes the blade is perfectly clamped, however there is some compliance in the bearings of the actual rotor stand.

In order to better compare the development of the natural frequencies with increasing rotational speed, the analytical flap bending frequencies were scaled according to the results of the rap test. The adjusted fan plot is shown in Figure 6.5. The experimental frequencies are compared with the adjusted analytical frequencies in Table 6.2.

\begin{tabular}{|c|c|c|c|c|c|}
\hline Mode & & $0 \mathrm{RPM}$ & $300 \mathrm{RPM}$ & $600 \mathrm{RPM}$ & $900 \mathrm{RPM}$ \\
\hline \multirow{3}{*}{$\begin{array}{l}\text { First } \\
\text { Flap }\end{array}$} & Analytical (Hz) & 13.6 & 14.4 & 15.6 & 20.9 \\
\hline & Experimental (Hz) & $13.6 \pm 0.01$ & $14.5 \pm 1.2$ & $14.9 \pm 0.8$ & $18.8 \pm 1.6$ \\
\hline & Percent Difference & $0.0 \%$ & $0.6 \%$ & $4.3 \%$ & $10.0 \%$ \\
\hline & & & & & \\
\hline \multirow{3}{*}{$\begin{array}{c}\text { Second } \\
\text { Flap }\end{array}$} & Analytical (Hz) & 81.6 & 82.4 & 84.2 & 89.4 \\
\hline & Experimental (Hz) & $81.9 \pm 0.01$ & $82.6 \pm 0.8$ & $83.1 \pm 0.6$ & $88.0 \pm 1.9$ \\
\hline & Percent Difference & $0.3 \%$ & $0.2 \%$ & $1.3 \%$ & $1.6 \%$ \\
\hline \multirow{4}{*}{$\begin{array}{l}\text { Third } \\
\text { Flap }\end{array}$} & & & & & \\
\hline & Analytical (Hz) & 212.8 & 213.6 & 216.1 & 221.0 \\
\hline & Experimental $(\mathrm{Hz})$ & $214.8 \pm 0.3$ & $195.5 \pm 12.7$ & $199.8 \pm 2.0$ & $209.7 \pm 1.9$ \\
\hline & Percent Difference & $0.9 \%$ & $8.5 \%$ & $7.5 \%$ & $5.1 \%$ \\
\hline
\end{tabular}

Table 6.2: Adjusted analytical vs. experimental natural frequencies. Analytical frequencies have been scaled to the results of the rap test. 


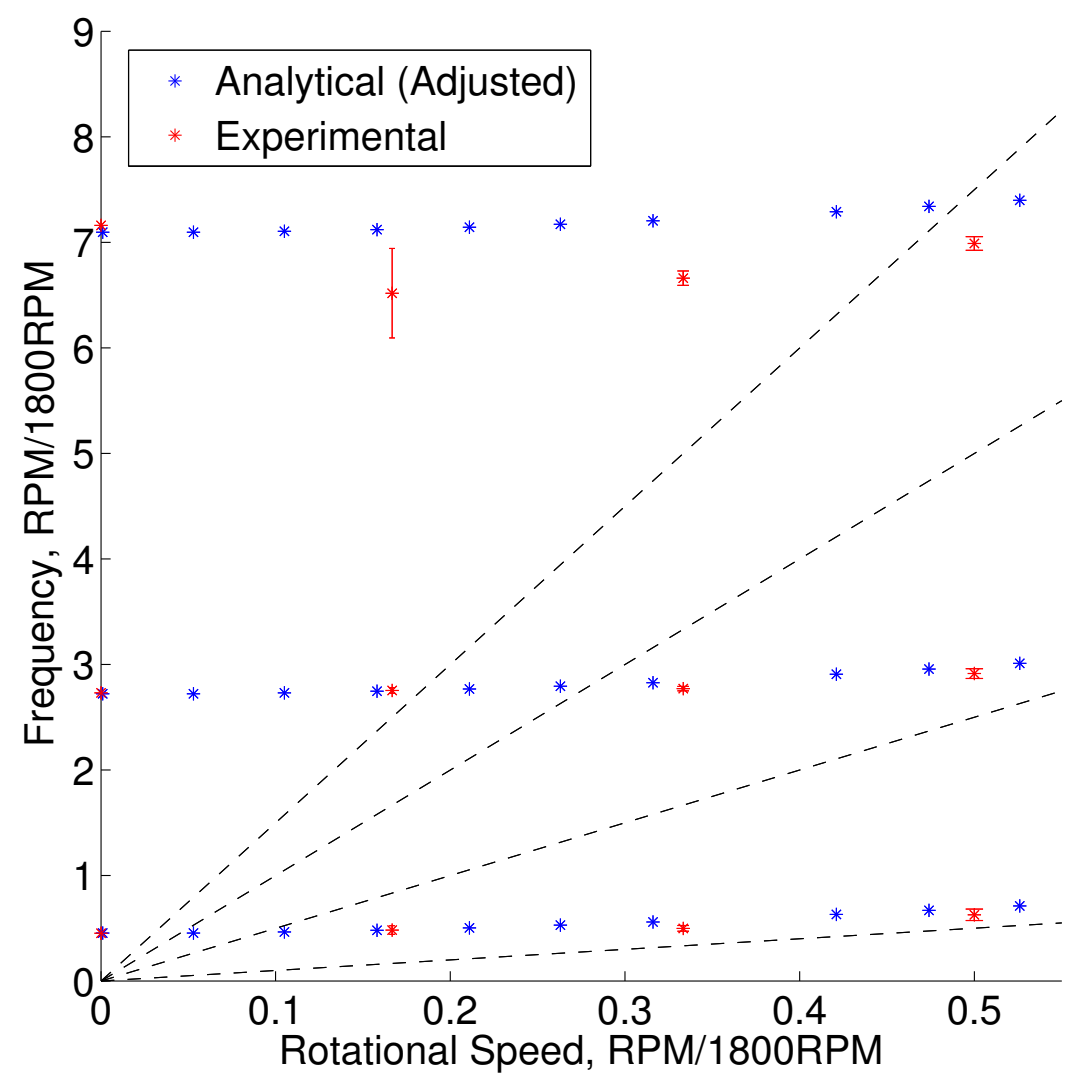

Figure 6.5: Natural frequencies vs. rotational speed. Analytical frequencies have been scaled according to the results of the rap test. Dashed lines correspond to one, five, ten, and fifteen per revolution frequencies. 
The experimentally determined natural frequencies agree with the scaled analytical natural frequencies. The second flap bending frequencies in particular agree within $2 \%$ of the analytical predictions for all rotational speeds. The first flap bending frequency at 900 RPM was the furthest from the predicted value with a $10 \%$ difference between the experimental and analytical natural frequencies. With an uncertainty of $\pm 8.5 \%$, it was also the least repeatable of the measurements. This is a result of the sampling frequency used to generate the response. At $900 \mathrm{RPM}$, the blade would move out of the field of view before enough images could be captured to formulate a response at a sampling rate of $500 \mathrm{~Hz}$, so the modal parameters were determined using a sampling rate of $1000 \mathrm{~Hz}$. As discussed in Section 4.2.0.3, if the sampling rate is greater than 100 times the frequency of interest, not enough modal displacement will occur between each time shifted response matrix to accurately determine the modal parameters. A sampling rate of $1000 \mathrm{~Hz}$ approaches this limit for the first flap bending mode, so it is not as well resolved as the mode shapes generated with a $500 \mathrm{~Hz}$ sampling rate, especially near the root.

The experimental natural frequencies also generally exhibit the expected trend of increasing natural frequency with increasing rotational speed. One notable discrepancy is the decrease in frequency of the third flap bending mode calculated by the ITD method between the non-rotating case and at 300 RPM. This unexpected result bears further investigation, especially as after the initial drop in frequency, the third mode then increases with frequency as predicted. It must be noted that for the rotating tests, the excitation was 
generally near the tip of the blade, which would not excite the third mode as strongly as the first two modes. Thus, the third mode was much more difficult to resolve using ITD analysis. In particular, the third mode at 300 RPM exhibited a large amount of scatter for different formulations of the response matrices as described in Section 5.7, and typically it would not pass the frequency matching and MSCC thresholds set to remove computational modes.

The first flap bending mode shape at each rotational speed overlaid on the analytical prediction is shown in Figure 6.6 through Figure 6.9.

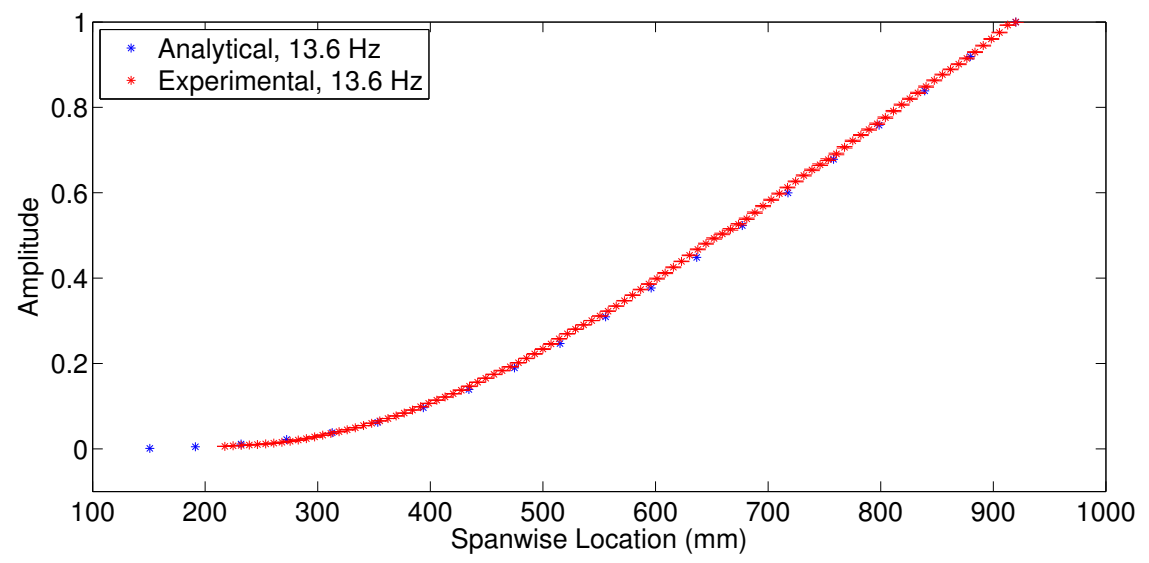

Figure 6.6: First flap bending mode shapes at 0 RPM. 


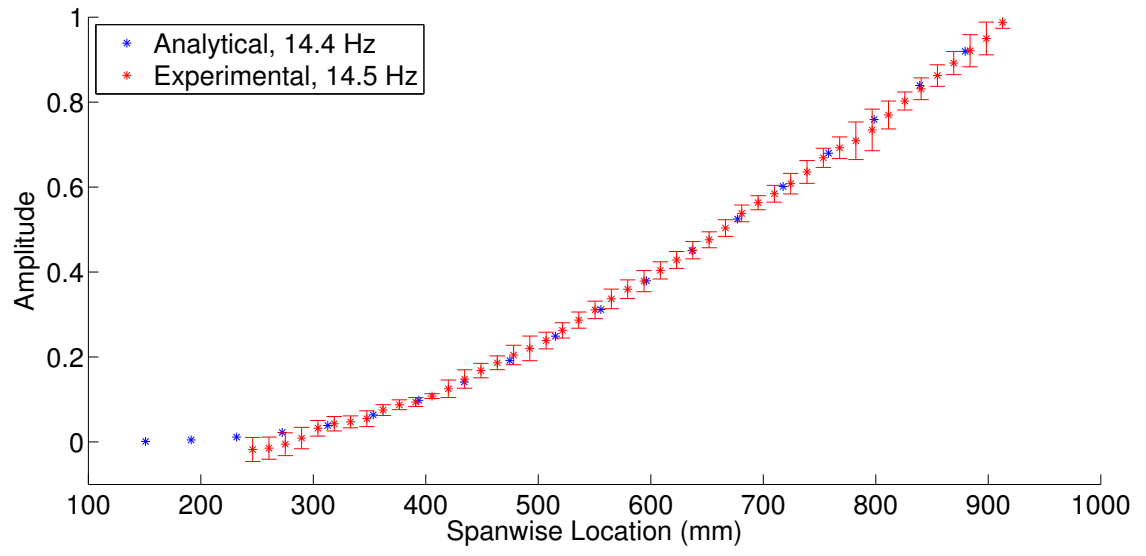

Figure 6.7: First flap bending mode shapes at 300 RPM.

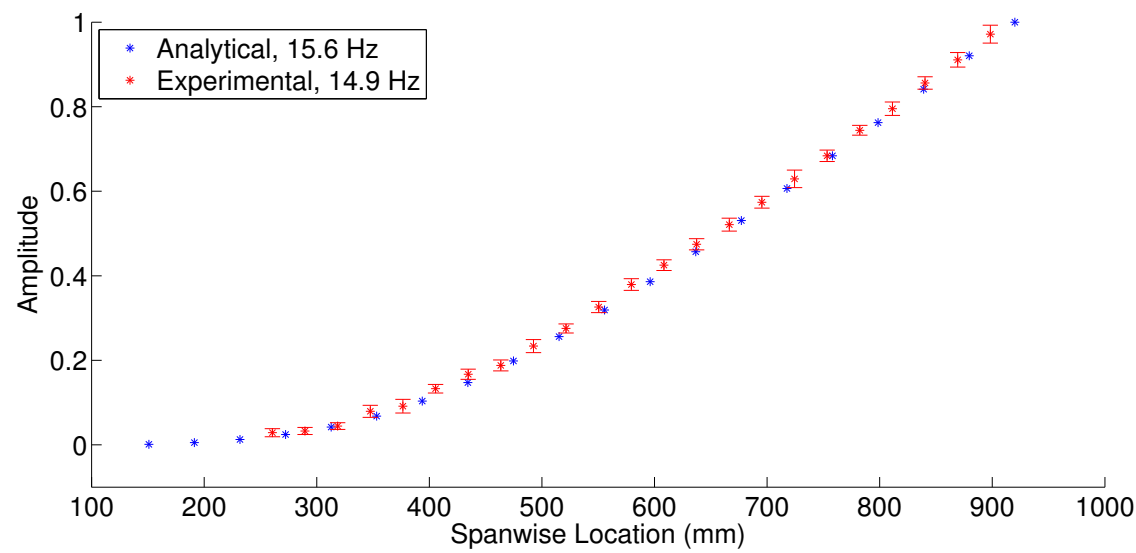

Figure 6.8: First flap bending mode shapes at 600 RPM. 


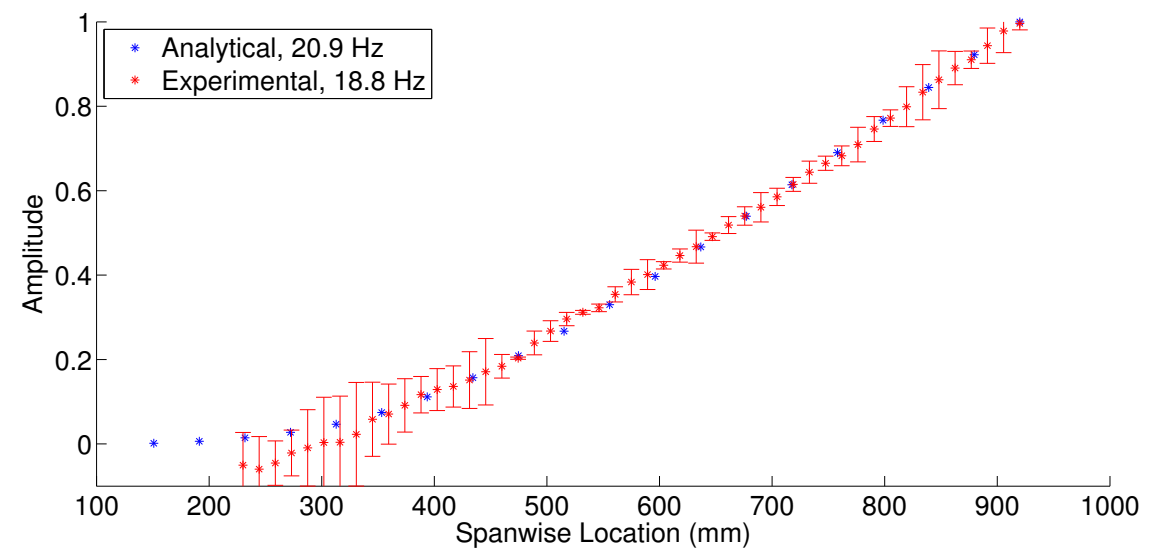

Figure 6.9: First flap bending mode shapes at 900 RPM.

The experimental first flap bending mode shapes closely follow the analytical prediction for all rotational speeds. The variance of the first mode shape at 900 RPM is greater than the other rotational speeds. Again, this is a result of the high sampling rate used to formulate the ITD analysis at 900 RPM.

The number of measurement locations along the span of the blade was varied to produce more mode shapes that could be averaged and statistically analyzed. If a formulation was used with a lower spatial resolution, only the measurement locations found in every formulation were collected and averaged. This prevented biasing the mode shapes in favor of the formulations with high spatial resolution.

The second flap bending mode shapes are shown in Figure 6.10. 

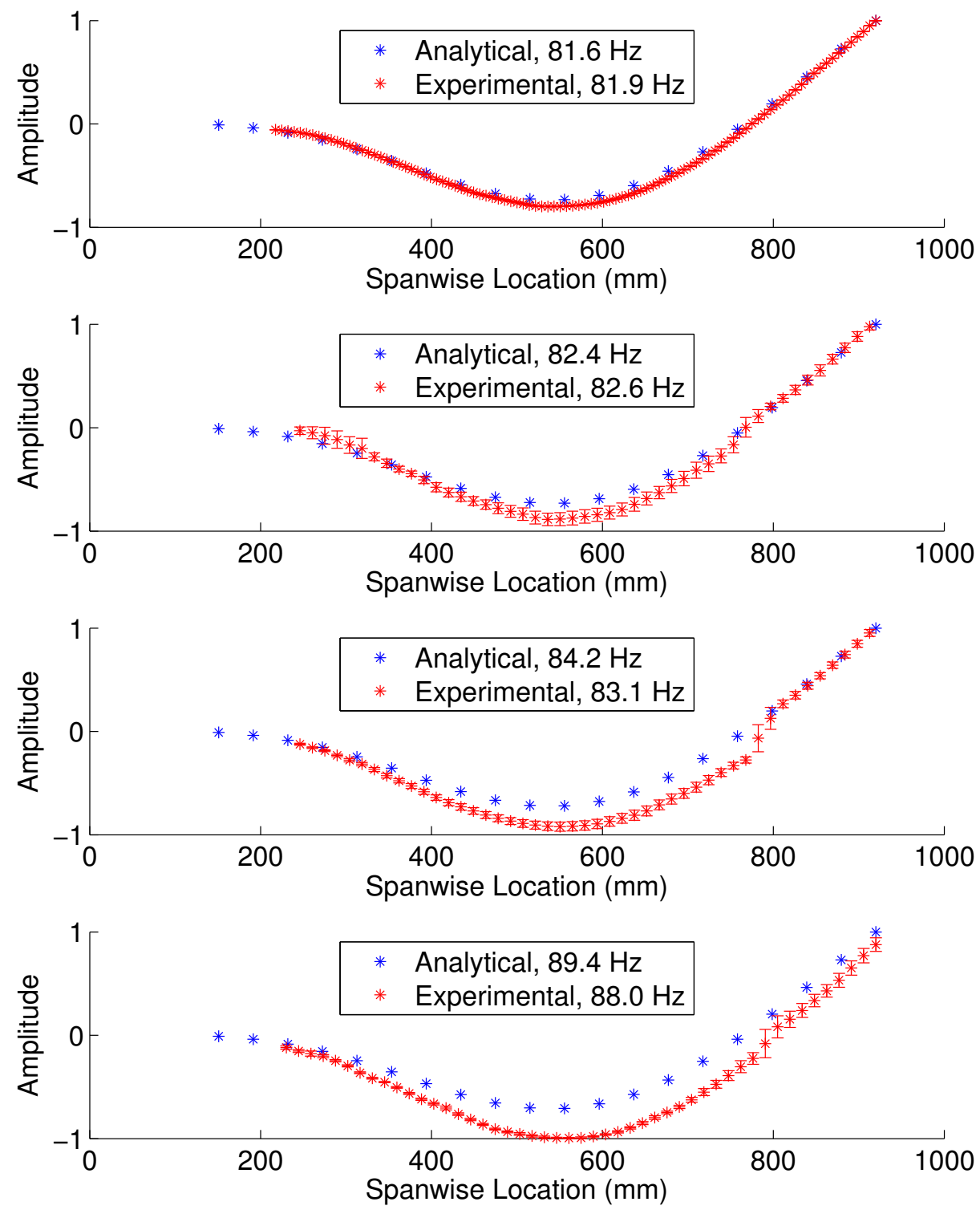

Figure 6.10: Second flap bending mode shapes. First plot is at 0 RPM, second is at $300 \mathrm{RPM}$, third is at $600 \mathrm{RPM}$, fourth is at $900 \mathrm{RPM}$ 
For every rotational speed, the second flap bending mode was the best resolved and most repeatable mode shape produced. It is therefore interesting to note that as the rotational speed increases the difference between the analytical and experimental mode shape increases.

The slight discontinuity in the mode shape at the node near the tip is an artifact of the spatial resolution and the method of approximating real-valued mode shapes from complex mode shapes. The magnitude of the complex shapes was plotted, and when a minimum was found, the sign of the amplitude was flipped. The slope of the shape is large at the location of the discontinuity, so if a minimum was found at slightly different locations for different solutions the resultant variation would be large.

The third flap bending mode shapes are shown in Figure 6.11. 

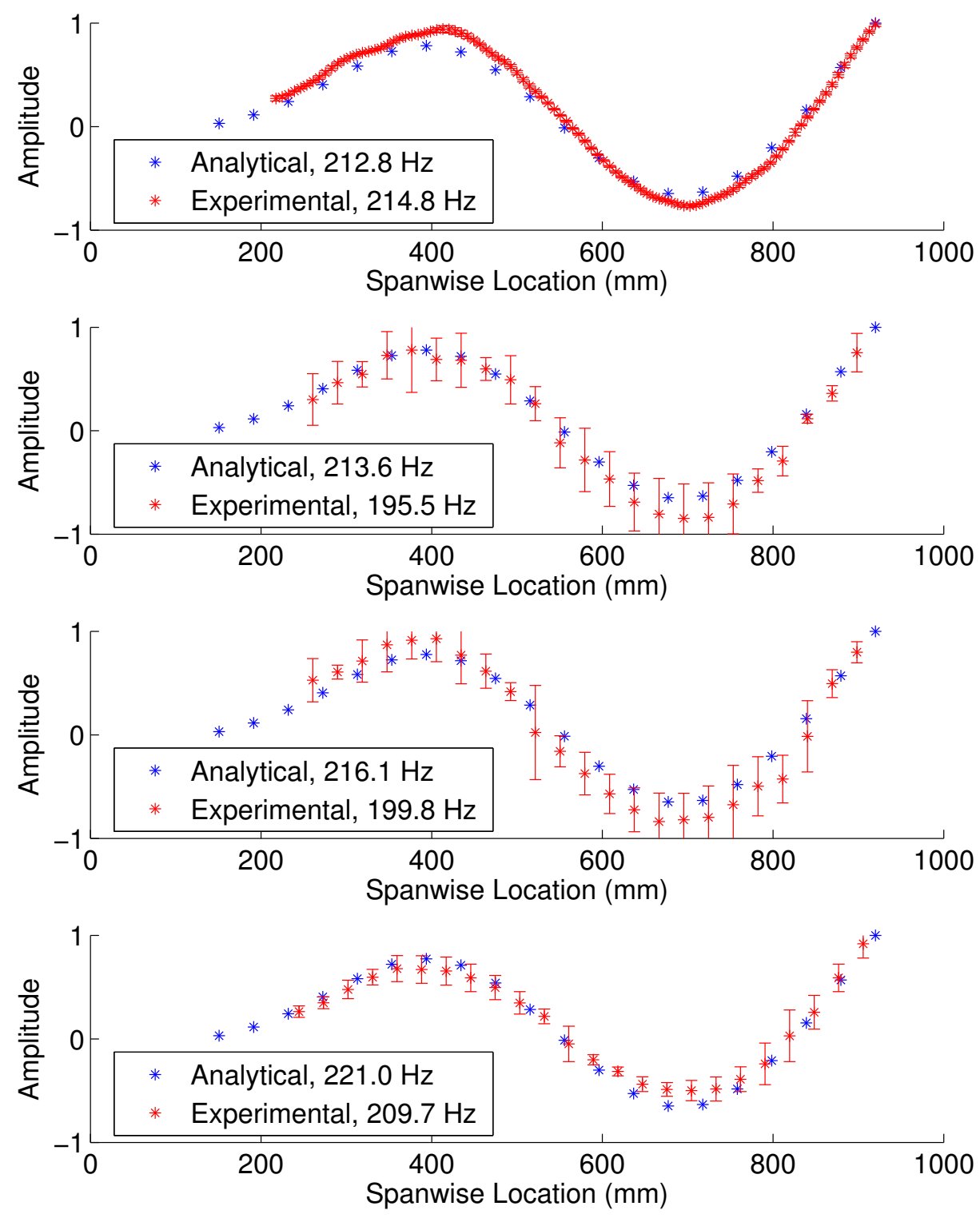

Figure 6.11: Third flap bending mode shapes. First plot is at 0 RPM, second is at $300 \mathrm{RPM}$, third is at $600 \mathrm{RPM}$, fourth is at $900 \mathrm{RPM}$ 
As previously discussed, the third mode shapes are not as well resolved as the other mode shapes for the rotating tests. This is primarily a result of the method of excitation. However, the experimental shapes still follow the analytical shapes well.

To better investigate the development of the mode shapes with increasing rotational speed, the mode shapes from the different tests were overlaid on one another. The first flap bending mode shapes are shown in Figure 6.12. 

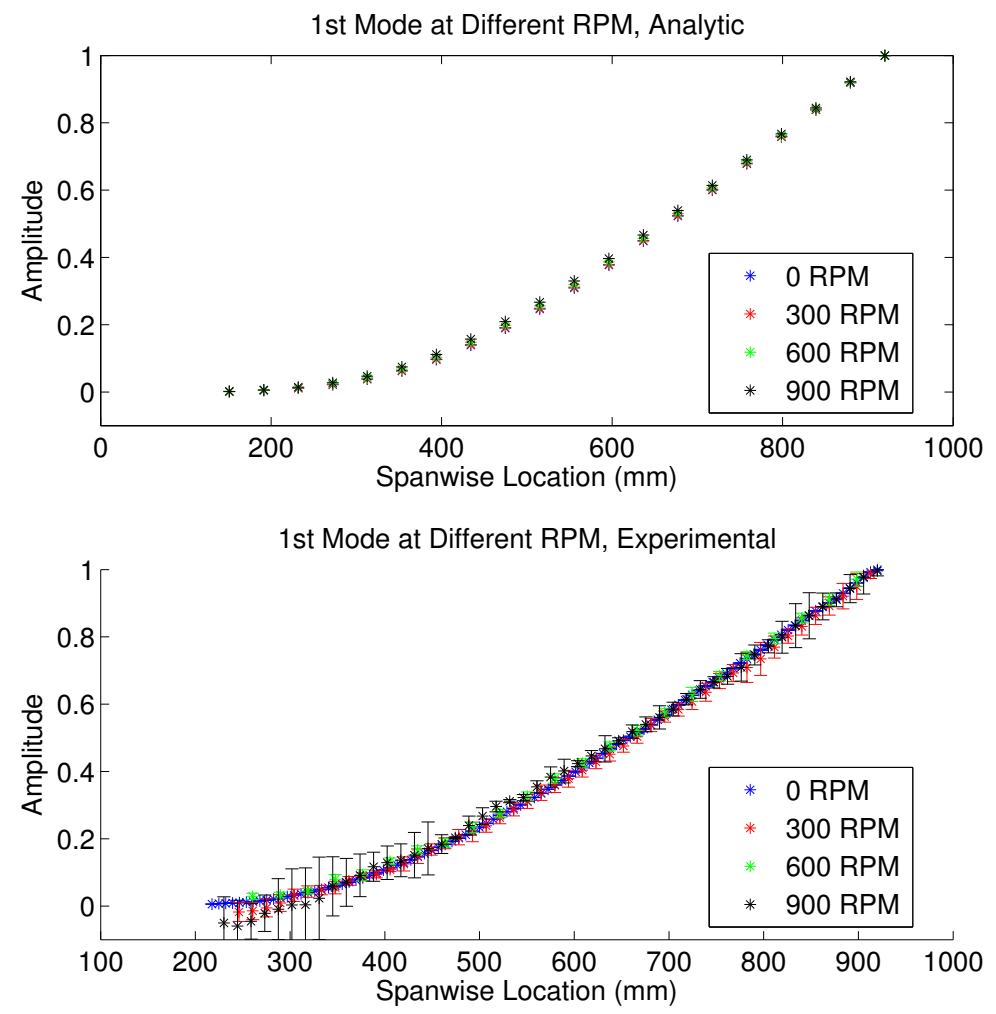

Figure 6.12: First flap bending mode shapes with increasing rotational speed. The upper plot shows the analytical mode shapes at 0, 300, 600, and 900 RPM. The lower plot shows the experimental mode shapes.

As expected, the analytical first flap bending mode tends to flatten out with increasing rotational speed, although the effect is small at these speeds. The variance of the experimental modes is too large to identify a trend.

The second flap bending mode shapes are shown in Figure 6.13. 

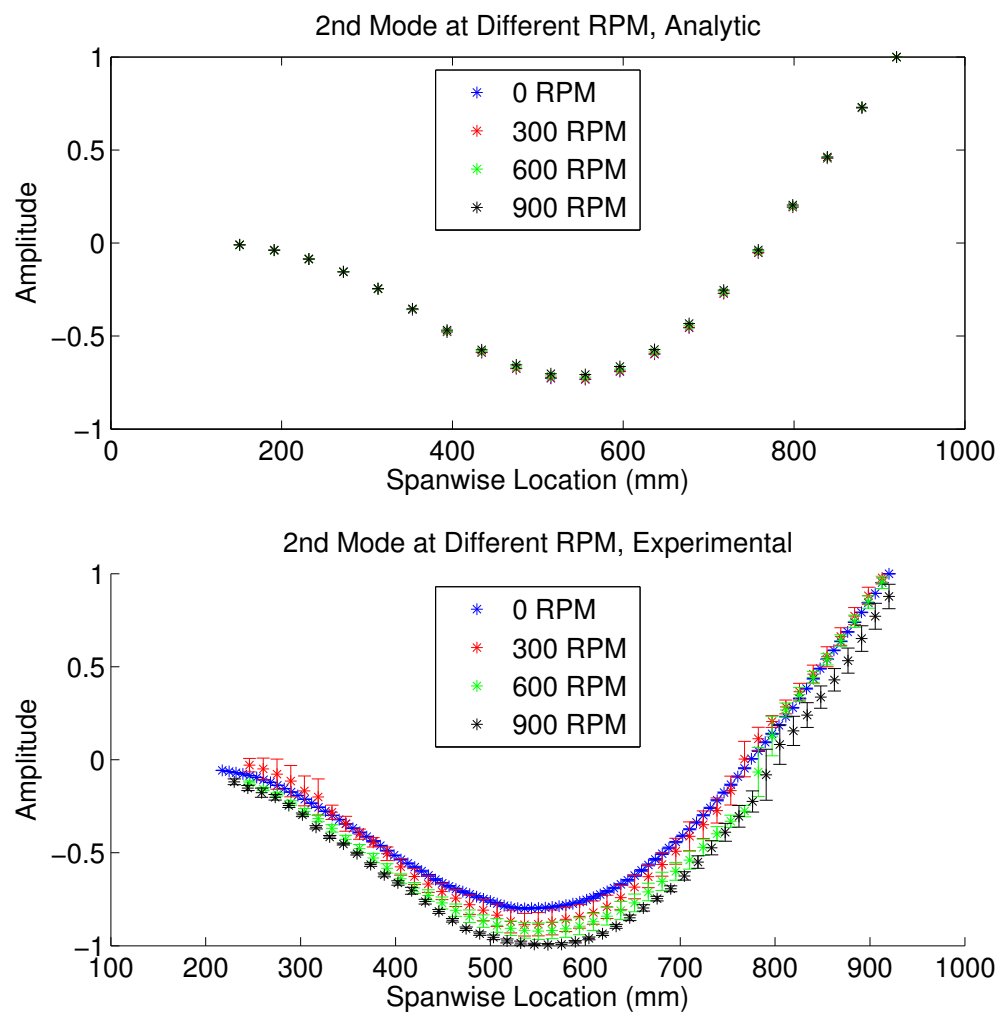

Figure 6.13: Second flap bending mode shapes.

The amplitude of the analytical second mode shapes near the midspan decreases with increasing rotational speed. The effect is even smaller than for the first mode. The trend of the experimental mode shapes is opposite of what was expected, however. With increasing rotational speed, the amplitude of vibration at the center of the beam apparently increases. As previously mentioned, the second mode shapes were the best resolved and most repeatable mode shapes produced, and the trend observed is quite distinct. Further experimentation is required to identify the cause of this trend. 
The third flap bending mode shapes are shown in Figure 6.14.
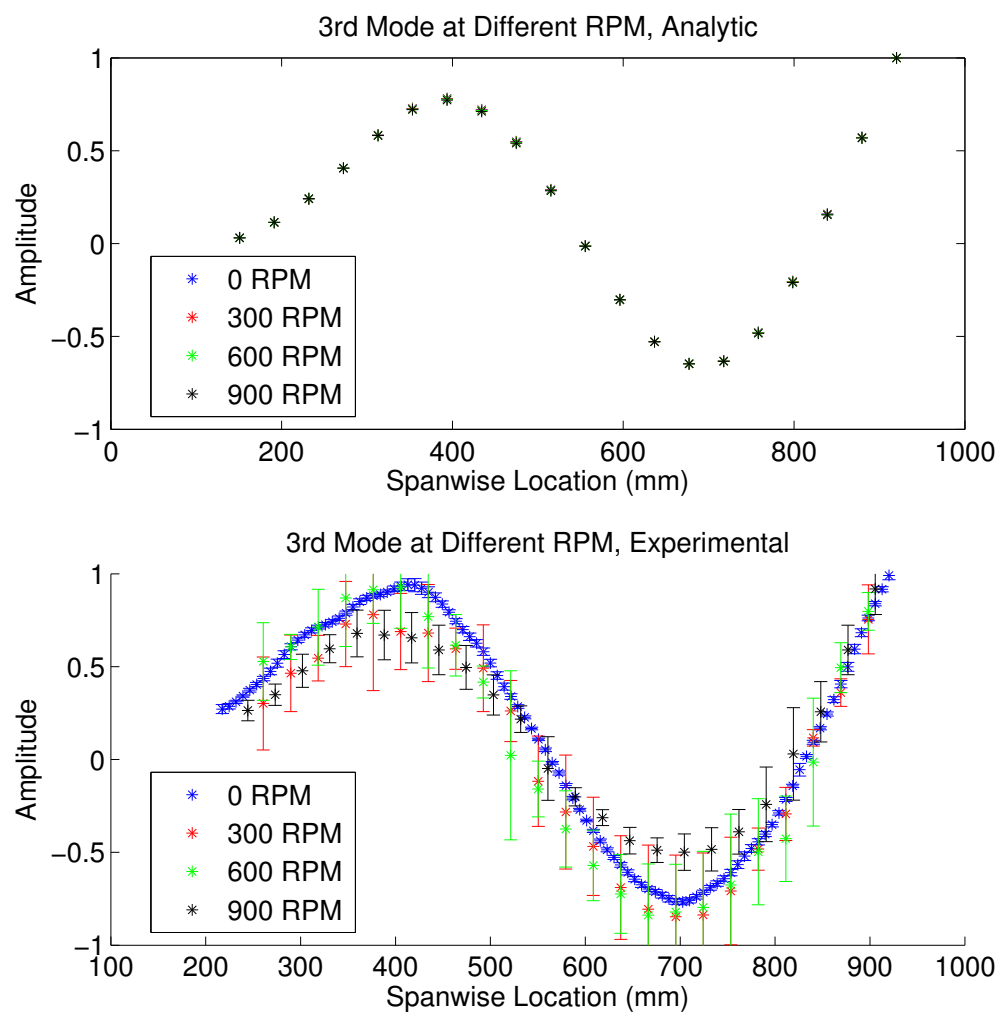

Figure 6.14: Third flap bending mode shapes.

There is almost no difference in the analytical mode shapes with increasing rotational speed. The large uncertainty in the experimental mode shapes make it difficult to determine a trend, however it appears that the inboard amplitude of the mode shape at 900 RPM is less than that of the non-rotating case.

As discussed in Section 4.3, the damping ratios calculated though ITD analysis are much less accurate than the natural frequencies and mode shapes. 
The damping ratios from this study are therefore not presented as part of the results, but are instead shown in Appendix A. 


\section{Chapter 7}

\section{Conclusion}

\subsection{Results Overview}

The first three flap bending natural frequencies and mode shapes of a rotating helicopter blade were experimentally determined over a range of 0 to 900 RPM and were compared to an analytical model of the system (finite element method). It was found that the analytical model over-predicted the natural frequencies due to differing boundary conditions between the model and the experiment. The analytical frequencies were scaled to the results of a rap test using traditional frequency domain analysis and compared to the experimental frequencies. The scaled analytical and experimental natural frequencies showed good agreement, especially for the non-rotating test and the 2 nd flap bending mode for the rotating test. The non-rotating frequencies matched to within $0.9 \%$, and the rotating 2 nd natural frequencies matched to within $1.6 \%$. The first flap bending mode at 900 RPM had the largest deviation

from the analytical value with a $10.0 \%$ difference between the frequencies. The third mode for rotating tests also deviated from the analytical prediction by $5.1 \%$ to $8.5 \%$.

The uncertainty of the natural frequencies ranged from $0.1 \%$ for the 
third mode at 0 RPM to $8.5 \%$ for the first mode at 900 RPM. The average uncertainty of the mode shapes ranged from $0.09 \%$ for the first mode at 0 RPM to $5.6 \%$ for the third mode at 600 RPM.

One notable unexpected result is an apparent decrease in the third flap bending natural frequency from non-rotating to rotating conditions. Another unexpected result is an increase in the amplitude of vibration at the midspan of the second flap bending mode with increasing rotational speed. Further investigation is required to definitively determine the cause of these results.

This body of work represents the first integration of DIC with ITD analysis. The results suggest that this operational modal analysis procedure is an effective method to experimentally determine the modal parameters of rotating systems.

\subsection{Research Recommendations}

While the results from this first attempt at rotational modal analysis using this procedure are promising, there are several aspects of both the data collection and analysis procedures that may be improved to increase the accuracy, repeatability, and functionality of the results. Recommendations for further research and development are as follow:

\section{Data Collection}

(a) Improve calibration procedure. The calibration used to produce the results in this analysis was performed using a single view of a $2 \mathrm{D}$ 
calibration plate. While this is sufficient to produce an acceptable mapping function, the calibration can be further improved by capturing multiple images of the plate at different heights and angles of tilt. An adjustable mount may be constructed to accomplish this.

(b) Provide stochastic excitation. The current method of excitation provides a single impulse per revolution. This produces a strong forcing mode that can be difficult to separate from the first flap bending mode. Developing a method to randomly excite the blade may alleviate this issue, although it will likely not eliminate it due to the cyclic nature of rotating systems. Activating and deactivating the air nozzle or translating it during the test should be investigated.

(c) Increase illumination and decrease exposure time to reduce tip blur. In order to perform DIC on the blade at higher rotational speeds, the camera exposure time must be decreased to reduce tip blur. This would require additional lighting. One proposed method is to speckle the test article with a fluorescent dye such as Rhodamine and illuminate it using a laser source.

(d) Increase field of view to capture more angular indices for response formulation. Capturing more images per revolution allows the user to artificially decrease the sampling rate by selecting every second, third, fourth, etc. time instant to analyze. This would allow the lower natural frequencies to be accurately identified at higher ro- 
tational speeds. The field of view can be increased by increasing the camera to test article distance, however this must be balanced against the ensuing loss of spatial resolution and accuracy. As was seen in the results of the second flap bending mode shape, a decreased spatial resolution may cause large variations in the mode shapes near the nodes.

(e) Improve test article preparation procedure. Over the course of this research project, a method to more accurately control the size and spacing of the speckles applied to the test article was sought. An attempt was made to define a speckle template using MATLAB, laser cut out the pattern on an acetate sheet, and spray paint over the template. However it was found that the edges of the produced speckles were not well defined. An improved speckling method would decrease test preparation time and allow patterns to be defined for different test cases.

\section{Analysis Procedure}

(a) Identify other modes. The current ITD analysis procedure can be extended to detect torsional, lead-lag, and stretching modes. This can be accomplished by changing the measurement locations used to formulate the ITD analysis. For example, the torsional modes may be found if two parallel strips of deformation vectors at the leading and trailing edges of the blade are analyzed. However, the accuracy 
of the measurements would need to be improved, as the magnitude of this deformation is smaller than the flap bending deformation.

(b) Improve identification of structural modes. As noted in Section 5.7, filters employing the MSCC and frequency matching occasionally would not remove all the computational modes characterizing the noise. Additionally, forcing modes at harmonics of the frequency of rotation are generally present in the solution and must be manually identified and excluded. Furthermore, the response due to random excitation is assumed to be negligible compared to the once-perrevolution excitation. A possible solution to some or all of these issues is proposed by Mohanty and Rixen [23]. They describe an algorithm combining ITD and Single Station Time Domain analysis methods that is capable of extracting modal parameters when harmonic forcing is present at a frequency known a-priori.

(c) Develop method to measure modal participation factors. One limitation of the ITD procedure is the inability to determine the relative contribution of each mode to the measured response. A possible approach to determine the modal participation factors is to scale the mode shapes according to the measured deflection shapes.

\subsection{Future Applications}

This analysis procedure may be used to develop and verify models of rotating systems such as helicopter blades and wind turbines. Current 
methods to experimentally determine the modal parameters of such systems under operating conditions are limited in number and functionality. Further development of this procedure can produce an effective, robust, and relatively simple non-contact tool to characterize rotating systems.

Another potential application of this procedure is in damage detection. There are a large number of studies of damage detection through modal analysis of static structures. For example, Zou et al.[24] explore the use of piezoelectric sensors and actuators to detect delamination in composite structures from the vibration response. Using ITD and DIC to perform damage detection offers a number of advantages over traditional methods. The ability of DIC to measure the full field deformation of a structure allows detection of localized strain concentrations that may be missed using sensors placed at discrete locations. Also, DIC is a non-contact method of measurement, so it will have no effect on the vibration response, unlike accelerometers or strain gages. Lastly, determining the modal parameters of a system in rotation may detect damage that would be missed in a static test. For example, if the properties of the mounting or bearing changed, the boundary conditions of the system in rotation would change, even though the boundary conditions of the non-rotating system may remain approximately the same. Thus, only the modal parameters of the system while rotating would be affected. 
Appendix 


\section{Appendix A}

\section{Experimental Damping Ratios}

\begin{tabular}{|c|c|}
\hline Mode & Damping Ratio \\
\hline First Flap & 0.0145 \\
\hline Second Flap & 0.0081 \\
\hline Third Flap & 0.0010 \\
\hline
\end{tabular}

Table A.1: Experimental damping ratios for cantilever beam experiment 


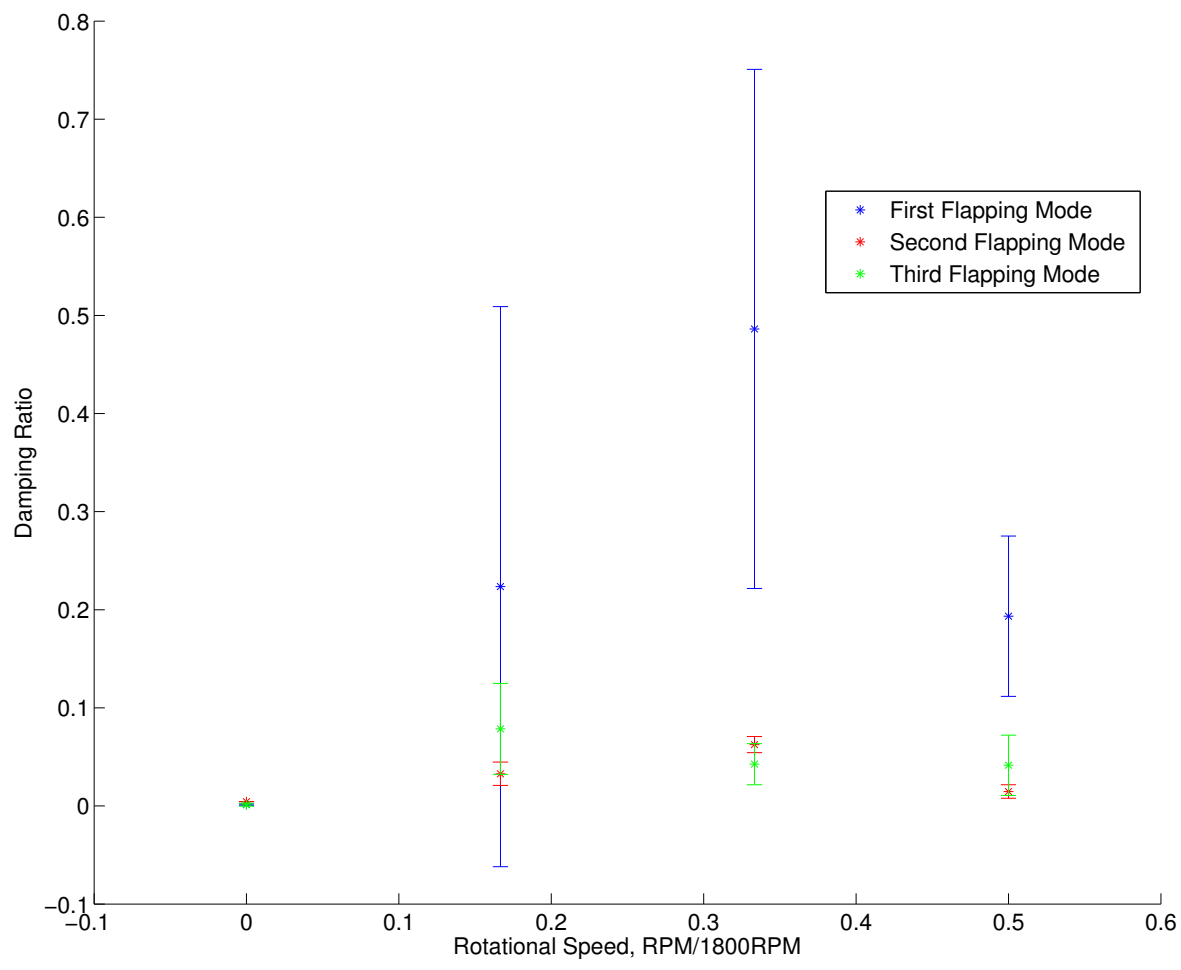

Figure A.1: Experimental damping ratios for helicopter blade experiment 


\section{Bibliography}

[1] Kane, T., Ryan, R., and Banerjee, A., "Dynamics of a cantilever beam attached to a moving base," Journal of Guidance, Control, and Dynamics, Vol. 10, No. 2, 1987, pp. 139-151.

[2] Banerjee, J., "Free vibration of centrifugally stiffened uniform and tapered beams using the dynamic stiffness method," Journal of Sound and Vibration, Vol. 233, No. 5, 2000, pp. 857-875.

[3] Rao, S. and Gupta, R., "Finite element vibration analysis of rotating Timoshenko beams," Journal of Sound and Vibration, Vol. 242, No. 1, 2001, pp. 103-124.

[4] Hoa, S., "Vibration of a rotating beam with tip mass," Journal of Sound and Vibration, Vol. 67, No. 3, 1979, pp. 369-381.

[5] Lien-Wen, C. and Chiung-Lu, C., "Vibration and stability of cracked thick rotating blades," Computers \& structures, Vol. 28, No. 1, 1988, pp. 67-74.

[6] Yoo, H. and Shin, S., "Vibration analysis of rotating cantilever beams," Journal of Sound and Vibration, Vol. 212, No. 5, 1998, pp. 807-828.

[7] Bucher, I. and Ewins, D., "Modal analysis and testing of rotating structures," Philosophical Transactions of the Royal Society of London. Series 
A: Mathematical, Physical and Engineering Sciences, Vol. 359, No. 1778, 2001, pp. 61-96.

[8] Wilkie, W. K., Mirick, P. H., Langston, C. W., and Command, U. A. M., Rotating shake test and modal analysis of a model helicopter rotor blade, National Aeronautics and Space Administration, Langley Research Center, 1997.

[9] Lundstrom, T., Baqersad, J., and Niezrecki, C., "Using high-speed stereophotogrammetry to collect operating data on a Robinson R44 Helicopter," Special Topics in Structural Dynamics, Volume 6, Springer, 2013, pp. 401-410.

[10] Schwarz, B. J. and Richardson, M. H., "Introduction to operating deflection shapes," CSI Reliability Week, Vol. 10, 1999, pp. 121-126.

[11] Kahn-Jetter, Z. and Chu, T., "Three-dimensional displacement measurements using digital image correlation and photogrammic analysis," $E x$ perimental Mechanics, Vol. 30, No. 1, 1990, pp. 10-16.

[12] Sirohi, J. and Lawson, M. S., "Measurement of helicopter rotor blade deformation using digital image correlation," Optical Engineering, Vol. 51, No. 4, 2012, pp. 043603-1.

[13] Sicard, J. and Sirohi, J., "Measurement of the deformation of an extremely flexible rotor blade using digital image correlation," Measurement Science and Technology, Vol. 24, No. 6, 2013, pp. 065203. 
[14] Tran, J., Sirohi, J., Gao, H., and Wei, M., "Reduced-order modeling of loads and deformation of a flexible flapping wing," AIAA 2015-0177, 56th AIAA/ASCE/AHS/ASC Structures, Structural Dynamics, and Materials Conference, Kissimmee, Florida, Jan. 5-9, 2015.

[15] Ibrahim, S. and Mikulcik, E., "A method for the direct identification of vibration parameters from the free response," Shock and Vibration Bulletin, Bulletin 47, 1977.

[16] Ibrahim, S. R. and Pappa, R. S., "Large modal survey testing using the Ibrahim time domain identification technique," Journal of Spacecraft and Rockets, Vol. 19, No. 5, 1982, pp. 459-465.

[17] Ibrahim, S. R., "Double Least Squares Approach for Use in Structural Modal Identification," AIAA Journal, Vol. 24, No. 3, 1986, pp. 499-503.

[18] LaVision, Göttingen, Germany, StrainMaster Manual DaVis 8.2, March 2014.

[19] Siebert, T., Becker, T., Spiltthof, K., Neumann, I., and Krupka, R., "High-speed digital image correlation: error estimations and applications," Optical Engineering, Vol. 46, No. 5, 2007, pp. 051004-051004.

[20] Schreier, H. W., Braasch, J. R., and Sutton, M. A., "Systematic errors in digital image correlation caused by intensity interpolation," Optical engineering, Vol. 39, No. 11, 2000, pp. 2915-2921. 
[21] Cameron, C. G., Uehara, D., and Sirohi, J., "Transient Hub Loads and Blade Deformation of a Mach-Scale Coaxial Rotor in Hover," AIAA 20150177, 56th AIAA/ASCE/AHS/ASC Structures, Structural Dynamics, and Materials Conference, Kissimmee, Florida, Jan. 5-9, 2015.

[22] Schmaus, J. and Chopra, I., "Performance and Loads Prediction for a High Advance Ratio Coaxial Rotor," AIAA 2015-0177, 56th AIAA/ASCE/AHS/ASC Structures, Structural Dynamics, and Materials Conference, Kissimmee, Florida, Jan. 5-9, 2015.

[23] Mohanty, P. and Rixen, D., "A modified Ibrahim time domain algorithm for operational modal analysis including harmonic excitation," Journal of Sound and Vibration, Vol. 275, No. 1, 2004, pp. 375-390.

[24] Zou, Y., Tong, L., and Steven, G., "Vibration-based model-dependent damage (delamination) identification and health monitoring for composite structures review," Journal of Sound and vibration, Vol. 230, No. 2, 2000, pp. $357-378$.

[25] Poozesh, P., Baqersad, J., Niezrecki, C., Harvey, E., and Yarala, R., "Fullfield inspection of a utility scale wind turbine blade using digital image correlation," CAMX, Orlando, Florida, Oct. 13-16, 2014.

[26] Asmussen, J., Brincker, R., and Ibrahim, S., "Modal analysis based on the random decrement transform," Fracture and Dynamics, Vol. R9821, No. 110, 1998. 
[27] Ibrahim, S. R., "Computation of normal modes from identified complex modes," AIAA Journal, Vol. 21, No. 3, 1983, pp. 446-451.

[28] Özdemir, Ö. and Kaya, M., "Flapwise bending vibration analysis of a rotating tapered cantilever Bernoulli-Euler beam by differential transform method," Journal of Sound and Vibration, Vol. 289, No. 1, 2006, pp. 413420. 